\title{
Time and Space Scales of Significant Wave Heights
}

\author{
JEAN TOURNADRE
}

Institut Français de Recherche pour l'Exploitation de la Mer, Centre de Brest, Plouzané, France

\begin{abstract}
This paper presents a study of the temporal and spatial variability of the significant wave height $\left(H_{\mathrm{s}}\right)$ based on stationarity and correlation (spectral) analyses. A sea state is defined as a stationary state of a piecewise stationary stochastic random wave process. Rupture detection, i.e., detection of abrupt changes in $H_{\mathrm{s}}$, is used to identify the stationary components of the wave process. A sea state is characterized by its energy (i.e., $H_{\mathrm{s}}$ ) and by its duration of stationarity (spatial analysis) and length of stationarity (temporal analysis). Intensive in situ measurements of $H_{\mathrm{s}}$ and Geosat radar altimeter data are used to study the temporal and spatial $H_{\mathrm{s}}$ scales at two locations, in the North Sea and in the equatorial Atlantic. The stationarity analysis leads to the following results; (1) duration and length show a great variability and are distributed according to exponential probability laws, (2) $H_{\mathrm{s}}$ is distributed according to a Gumbel probability law in both time and space, (3) energy and duration and energy and length can be considered as statistically independent, and (4) the duration and length distributions present very similar nondimensional statistical characteristics. Stationary state detection can also be used to filter the high-frequency geophysical and/or instrumental noise from the $H_{\mathrm{s}}$ variations. A spectral analysis is performed on the raw $H_{\mathrm{s}}$, the stationary states, and the residual. The salient features of the results are summarized as follows: (1) for both locations, the spectra of the filtered data are consistent with a power law dependence on the wavenumber or frequency, (2) the spectral dependence is nearly the same for time and space, which suggests a mean linear dispersion relation for $H_{\mathrm{s}}$, (3) the slope of the spectra are close to the $-5 / 3$ turbulence cascade $(-1.74$ for the North Sea, -1.69 for the equatorial Atlantic), and (4) the residual spectrum is nearly a white noise spectrum indicating the quality of the stationary state detection filtering.
\end{abstract}

\section{INTRODUCTION}

The estimate of temporal and spatial variability of the mesoscale significant wave height $\left(H_{\mathrm{s}}\right)$ (1 hour to 3 days, 30 to $\left.100 \mathrm{~km}\right)$ is critical not only for many studies of oceanic and atmospheric processes but also in the calibration/validation process of satelliteborne wave sensors as well as for the prediction of extreme values of environmental parameters. These temporal and spatial scales are necessary, when calibrating a wave sensor, to estimate the effects of the spatial and temporal separations between in situ and satellite measurements [Monaldo 1988, 1990; Challenor et al., 1986]. Labeyrie $[1990,1991]$ showed that they also have to be taken into account when predicting extreme values of $H_{\mathrm{s}}$ from in situ data sets. Monaldo [1988, 1990], using spectral and correlation analysis of Geosat and Seasat altimeter measurements and buoy data, estimated the rms differences that can be expected from separation in time and space of two $H_{\mathrm{s}}$ measurements. Challenor et al. [1986] showed that the $H_{s}$ variations in both time and space can be separated into two components: a low-frequency part, associated with meteorological synoptic events, and a high-frequency part. Using cubic spline functions to fit the low-frequency signal, they showed that the high-frequency can be treated as instrumental and/or geophysical noise.

These two studies are based on correlation analysis, and as Challenor et al. [1986] stated, correlations are not easy to interpret in terms of dominant scales. Correlation analysis assumes that the data are statistically stationary, which is generally true [Challenor, 1983]. However, there are short distances over which the statistical characteristics of the wave process change rapidly and where the stationarity hypothesis does not hold [Queffeulou, 1983; Challenor of al., 1986]. An ideal scale analysis should combine stationarity and correlation analyses.

Copyright 1993 by the American Geophysical Union.

Paper number $92 \mathrm{JC} 02625$.

$0148-0227 / 93 / 92 \mathrm{JC}-02625 \$ 05.00$
Methods have been developed to identify stationary states within stochastic random processes [Labeyrie, 1990, 1991; Lavielle and Rio, 1987]. They have been used to improve short- and longterm extreme value prediction from in situ $H_{\mathrm{s}}$ measurements [ $\mathrm{La}$ beyrie, 1990]. This paper presents a scale analysis in both time and space of the $H_{\mathrm{s}}$ variations based on stationarity and correlation analyses.

In situ data from acquisition systems set up on two platforms, one in the Frigg oil field $\left(60^{\circ} \mathrm{N}, 2^{\circ} \mathrm{E}\right.$, North Sea) and the other in the Palanca field $\left(6^{\circ} \mathrm{S}, 12^{\circ} 30^{\prime} \mathrm{E}\right.$, Angola coast) are used to study the time scales. Altimeter measurements from the Geosat satellite are used to study the space scales. Section 2 presents the significant wave height data sets used in the study. The method of identification of stationary states is then discussed in section 3 , then in section 4 it is applied to the in situ and altimeter $H_{\mathrm{s}}$ data sets to construct sequences of stationary (in time or space) sea states at the two different locations. The stationary state data sets are analyzed statistically in terms of dominant space and time scales in section 4. Section 5 presents the results of the spectral analysis of the raw $H_{\text {s }}$ measurements, the stationary states and the residual. In the final section the results are summarized and analyzed.

\section{Signimcant Wave Height Dhta Set}

\subsection{Geosat Data}

The U.S. Navy Geosat satellite was launched in March 1985 and used a radar altimeter to estimate wind speed and significant wave height. The altimeter is a $13.5-\mathrm{GHz}$ nadir-pointing radar that measures the range between the satellite and the ocean surface. Measurements of the return signal give the backscatter coefficient, and the received power as a function of time. The slope of the front edge of the received power signature is a function of the $H_{\mathrm{s}}$ [Brown, 1979; Fedor and Brown, 1982; McArthur et al., 1987]. The instrument was designed to measure $H_{\mathrm{s}}$ with a precision of $10 \%$ or $0.5 \mathrm{~m}$ (whichever is greater). $H_{\mathrm{s}}$ is computed on board via 
an algorithm documented by Cole and May [1985]. The altimeter provides a $H_{\mathrm{s}}$ estimate every second, or every $7 \mathrm{~km}$, along the satellite ground track.

During the first 18 months after its launch, Geosat operated in a geodetic mapping mode. Then, on October 1, 1986, the satellite was put into a 17-day exact repeat orbit optimized for collecting oceanographic data. This constitutes the Exact Repeat Mission (ERM), which ended with the radar's "death" in September 1989.

\subsection{In Situ Data}

Data from radar distancemeter measurements made from the quarter platform of the Frigg field and from a platform of the Palanca field are used extensively. The first platform (named Frigg hereinafter) is located in the North Sea between the Shetland Islands and Norway $\left(60^{\circ} \mathrm{N}, 2^{\circ} \mathrm{E}\right)$ in nearly $100-\mathrm{m}$ water depth. Palanca is located off the coast of Angola in the equatorial Atlantic $\left(6^{\circ} \mathrm{S}\right.$, $12^{\circ} \mathrm{E}$ ). The water depth is $47 \mathrm{~m}$. The measurements are under the responsibility of the platforms' operator, the Elf company. The seasurface elevation data are recorded at a $2-\mathrm{Hz}$ sampling frequency [Elf Aquitaine Norge, 1981]. Continuous data have been collected from January 1, 1984 to December 12, 1984 at Frigg and from November 11,1984 to November 11, 1985 at Palanca. These data have been used to compute $H_{\mathrm{s}}$ estimates from the variance of the 20-min-long sea-surface elevation data records.

\section{DETECTION of STATIONARY STATES}

Lavielle and Rio [1987], Basseville [1988], De Cambry and Labeyrie [1985], and Labeyrie [1990] have proposed methods of identifying of stationary states in stochastic processes. The methods that they proposed are based on the detection of ruptures or abrupt changes in the signal. The method presented by Lavielle and Rio [1987] and Labeyrie [1990] is used in this study. A detailed description is given in the papers by these authors and will thus only be outlined here.

The sea-surface elevation is described as a stochastic process of the form $X(t, A, \omega)$, where $t$ is time, $A$ is the location, and $\omega$ is a random argument. $X$ is assumed to be ergodic; i.e., the statistical measures attained by averaging a set of realizations at a given time (or location) are weakly equivalent to averaging a single realization for a sufficiently long interval of time (or space). Stationarity is considered here in the broad sense (or weakly stationary); i.e., a process is said to be stationary if its mean value is a constant and its correlation function is dependent only on the temporal (or spaíial) displacement [cf. Bendat and Piersol, 1971]. For spatial analysis, homogeneity should be used instead of stationarity; however, to simplify the text, stationarity will be used hereinafter for both analyses.

A sea state is defined as a stationary state of the piecewise stationary stochastic random wave process $X$. The significant wave height $H_{\mathrm{s}}$ is used to described the magnitude of the sea state. If $X$ has a zero mean, $H_{\mathrm{s}}$ is closely related to the variance of the seasurface elevation $m_{0}=E\left(X^{2}\right)$, i.e., to the potential energy of the sea-surface. For example, $H_{s}=4 \sqrt{m_{0}}$ if $\mathrm{X}$ is assumed to be a zero-mean, narrow-band, Gaussian process [Longuet-Higgins, 1952].

A method to identify in time (or space) the stationary component of the sea-surface elevation is to detect changes in $H_{\mathrm{s}} . H_{\mathrm{s}}$ can be estimated from in situ measurements (buoy, distance meter, etc.) or from satellite borne radar altimeter measurements [Brown, 1979]. $H_{\mathrm{s}}$ is computed from 20 -min-long in situ measurements of sea surface elevation (or pitch and roll). An altimeter provides an estimate of $H_{\mathrm{s}}$ every $7 \mathrm{~km}$ (or every second) along the satellite ground track. For a typical wave period of 7-9 s, $15-20 \mathrm{~min}$ in time are roughly equivalent to $7 \mathrm{~km}$ in space [Challenor et al., 1986; Challenor, 1983]. Comparisons of Geosat altimeter-inferred estimates of $H_{s}$ with coincident in situ measurements done at the Frigg platform $\left(60^{\circ} \mathrm{N}, 2^{\circ} \mathrm{E}\right)$ yield a mean difference of $0.2 \mathrm{~m}$ with a standard deviation of $0.47 \mathrm{~m}$ [Tournadre and Ezraty, 1990]. In a first-order approximation, Geosat data and 20 -min sea surface elevation data can be considered as equivalent. A sea state is characterized by a mean value of $H_{s t a t}$ and a duration of stationarity $\left(T_{\text {star }}\right)$ or a length of stationarity $\left(L_{\text {sial }}\right)$.

Let $H_{\mathrm{s}}(i)$ be a sequence of $H_{\mathrm{s}}$ estimates (spatial or temporal). Labeyrie [1990] proposed a method of detection of abrupt changes in the discrete process $H_{\mathrm{s}}$. As the process is stationary, there exists a constant value $H_{\text {stat }}$ such that

$$
H_{s}(i)=H_{s t a t}+\varepsilon(i)
$$

where the $\varepsilon(i)$ are indepenclent random noise samples whose distribution is Gaussian $N\left(0, \sigma^{2}\right)$ (hypothesis $\left.H_{0}\right)$. A sea state change is mainly due to a significant change in the mean of $\varepsilon(i)$. After a sea state change, $\varepsilon(i)$ is $N\left(\mu, \sigma^{2}\right)$ distributed (hypothesis $H_{1}$ ), assuming the variation of $\sigma$ to be negligible. The noise parameters $\mu$ and $\sigma$ describing the noise under the normal distribution assumption depend on measurement and statistical estimate uncertainties; $\mu$ is taken in the form $a H_{\text {stat }}+b$ and the results are proved robust both in time and space when simulating reasonable variations of $a$ and $b$ [Lavielle and Rio, 1987; Tournadre and Robin, 1990], and $\sigma$ is of the form $\alpha H_{\text {stat }}+\beta$, where $\alpha$ and $\beta$ are iteratively estimated. This estimate of the variance has been chosen because the classical estimator, $\hat{\sigma}$

$$
\hat{\sigma}=\sqrt{\frac{1}{n-1} \sum_{i=1}^{n}\left(H_{i}-\bar{H}\right)^{2}}
$$

might fail for low sea states.

The sequential detection algorithm is based on Hinkley's test [Willsky and Jones, 1976). The log-likelihood ratio process ( $\Psi$ ) is used as a statistic for the test. Let $m_{0}$ and $m_{1}$ be the likelihood of $\varepsilon$ under the $H_{0}$ and $H_{1}$ hypotheses, $\Psi$ is given by

$$
\Psi(\varepsilon)=\log \left(\frac{m_{1}(\varepsilon)}{m_{0}(\varepsilon)}\right)
$$

which becomes

$$
\Psi(\varepsilon)=\frac{\mu}{\sigma^{2}}\left(\varepsilon-\frac{\mu}{2}\right)
$$

because $\varepsilon$ is normally distributed. Two statistical series $X_{i}^{1}(\mu>0)$ and $X_{i}^{2}(\mu<0)$ are computed in parallel to analyze either increasing or decreasing variations from $H_{\text {stat }}$. The $X^{1}$ series is defined by

$$
\begin{gathered}
X_{0}^{1}=0 \\
X_{n+1}^{1}=\operatorname{Sup}\left(X_{n}^{1}+\Psi\left(\varepsilon_{n+1}\right), 0\right)
\end{gathered}
$$

where Sup is defined by

$$
\begin{array}{ll}
\operatorname{Sup}(x, y)=x & x>y \\
\operatorname{Sup}(x, y)=y & x<y
\end{array}
$$

The $X^{2}$ series is defined in the same manner for $\mu<0$. The $H_{1}$ hypothesis is accepted when $X_{n}^{1}$ (or $X_{n}^{2}$ ) is greater than a given 


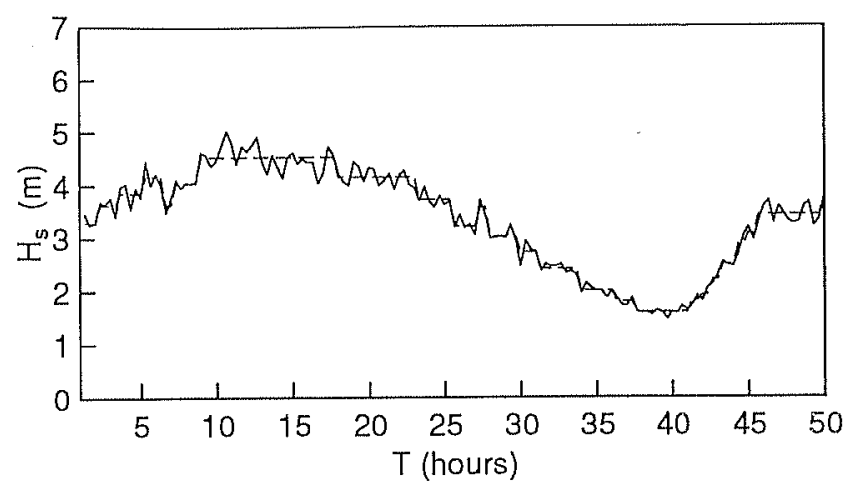

Fig. 1. Example of stationary state detection. Raw $H_{s}$ in situ measurement from the Frigg platform, solid line; stationary states $H_{\text {stat }}$, dashed line.

threshold. This threshold, $S$, has been evaluated to a value of 7 to 9 from a sensitivity study [Lavielle and Rio, 1987] in order to jointly minimize the probability of false alarm and the delay of detection (For more details about the theoretical law of the detector, see also Basseville and Benveniste [1983]).

Figure 1 shows an example of stationary state detection for a sequence of $H_{\mathrm{s}}$ in situ measurements at Frigg. The detection algorithm has been applied to time series of $H_{\mathrm{s}}$ estimates from in situ measurements of sea surface elevations and to space series from the Geosat radar altimeter. Two locations, Frigg and Palanca (for which continuous in situ measurements, one every $20 \mathrm{~min}$, are available), were considered. For each location, sequences of stationary sea states were constructed from the times series and from the measurements along the Geosat track located within a zone surrounding the point.

\section{STATIONARTTY ANALYSIS}

The main problem of the detection algorithm is the choice of the estimators of the noise parameters $\sigma$ and $\mu$. As was stated previously, the $\alpha$ and $\beta$ are iteratively estimated. A first estimate is given by the linear regression coefficients of the $H_{\mathrm{s}}$ standard deviation versus $H_{\mathrm{s}}$, where the standard deviation is computed from 20-sample-long running records of $H_{\mathrm{s}}$. The stationary states are then used to compute the standard deviation $\sigma$ of the $\varepsilon(i)$ (see equation (1)). A linear regression of $\sigma$ versus $H_{\text {stat }}$ gives the new estimates of $\alpha$ and $\beta$. The method converges in two or three iterations. The variations of $H_{\text {stat }}$ increase with $H_{\text {stat }}$ [Lavielle and Rio, 1987; Tournadre and Robin, 1990]. The $a$ and $b$ coefficients are first fixed a priori so that $\mu$ is between $0.20 \mathrm{~m}$ and $0.50 \mathrm{~m}$. The results of the detection algorithm are then used to validate the hypothesis and to adjust the coefficients. For each data set, the results are robust when simulating reasonable variations of the coefficients [Tournadre and Robin, 1990].

Table 1 summarizes the different noise parameters used for the four different $H_{\mathrm{s}}$ data sets: the Frigg in situ and altimeter data sets and the Palanca in situ and altimeter data sets. It can be seen in Table 1 that the noise parameters are very similar. However, it appears that the noise level of the $H_{\mathrm{s}}$ altimeter measurements is higher than that for the in situ measurements. This is especially true for low sea states $(<1.5 \mathrm{~m})$. This can be easily seen in Figure 2 , which presents the standard deviation of $H_{\mathrm{s}}$ (computed by running mean on 20 consecutive samples) as a function of $H_{\mathrm{s}}$ for both the Frigg in situ and altimeter data sets. Furthermore, for altimeter estimates, the noise level is higher for sea states below $1.5 \mathrm{~m}$ than for sea states between $1.5 \mathrm{~m}$ and $3 \mathrm{~m}$. This behavior of the $H_{\mathrm{s}}$ noise is mainly instrumental.
TABLE 1. Noise Parameters of the Stationary State Detection Algorithm used for the four Data Sets

\begin{tabular}{lccccc}
\hline Data Set & $a, \%$ & $b, \mathrm{~m}$ & $\alpha, \%$ & $\beta, \mathrm{m}$ & $\mathrm{S}$ \\
\hline Frigg in Situ & 10 & 0.05 & 4 & -0.01 & 7 \\
Frigg Satellite & 10 & 0.20 & 3 & 0.06 & 7 \\
Palanca in Situ & 10 & 0.05 & 3 & 0.02 & 7 \\
Palanca Satellite & 10 & 0.20 & 3 & 0.06 & 9 \\
\hline
\end{tabular}

$S$ is the threshold used for the detection (see texl).

This noise behavior can cause an instability of the detection algorithm. Figure 3 presents the log-likelihood process $\Psi$ used for the detection as a function of the noise $(\varepsilon)$ for four different values of $H_{\mathrm{s}}$ (Figure $3 a$ ) and as a function of $H_{\mathrm{s}}$ for six different $\varepsilon$ (Figure $3 b$ ). If one considers a low sea state $(<1.5 \mathrm{~m})$, since the noise
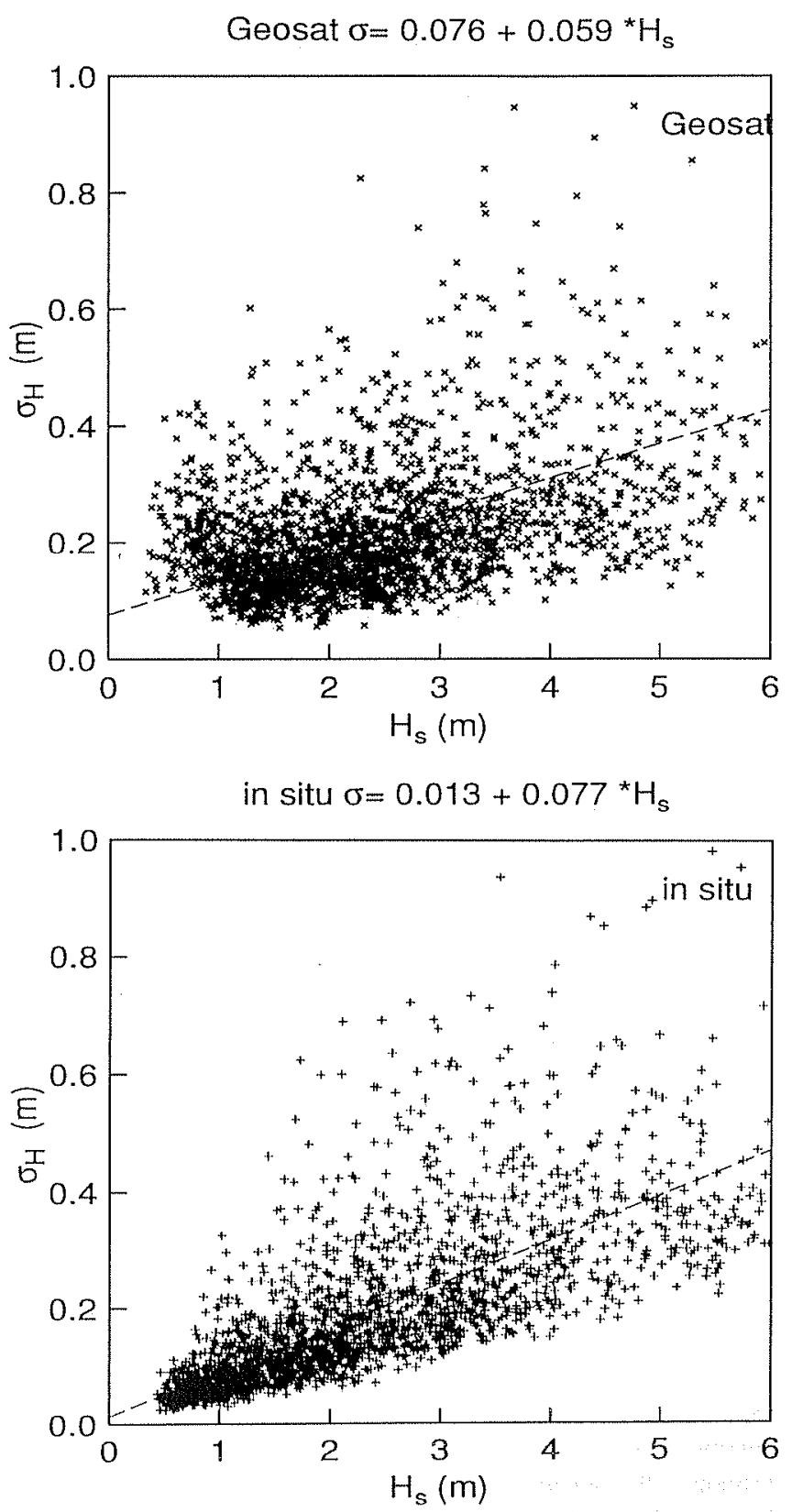

Fig. 2. Scatterplot of the variance $\sigma_{H}$ as a function of $H_{s}$. The variance is estimated from 20 sample running records for (bottom) in situ data and (top) satellite daa. The dashed lines indicate the nean square fit of the data. 

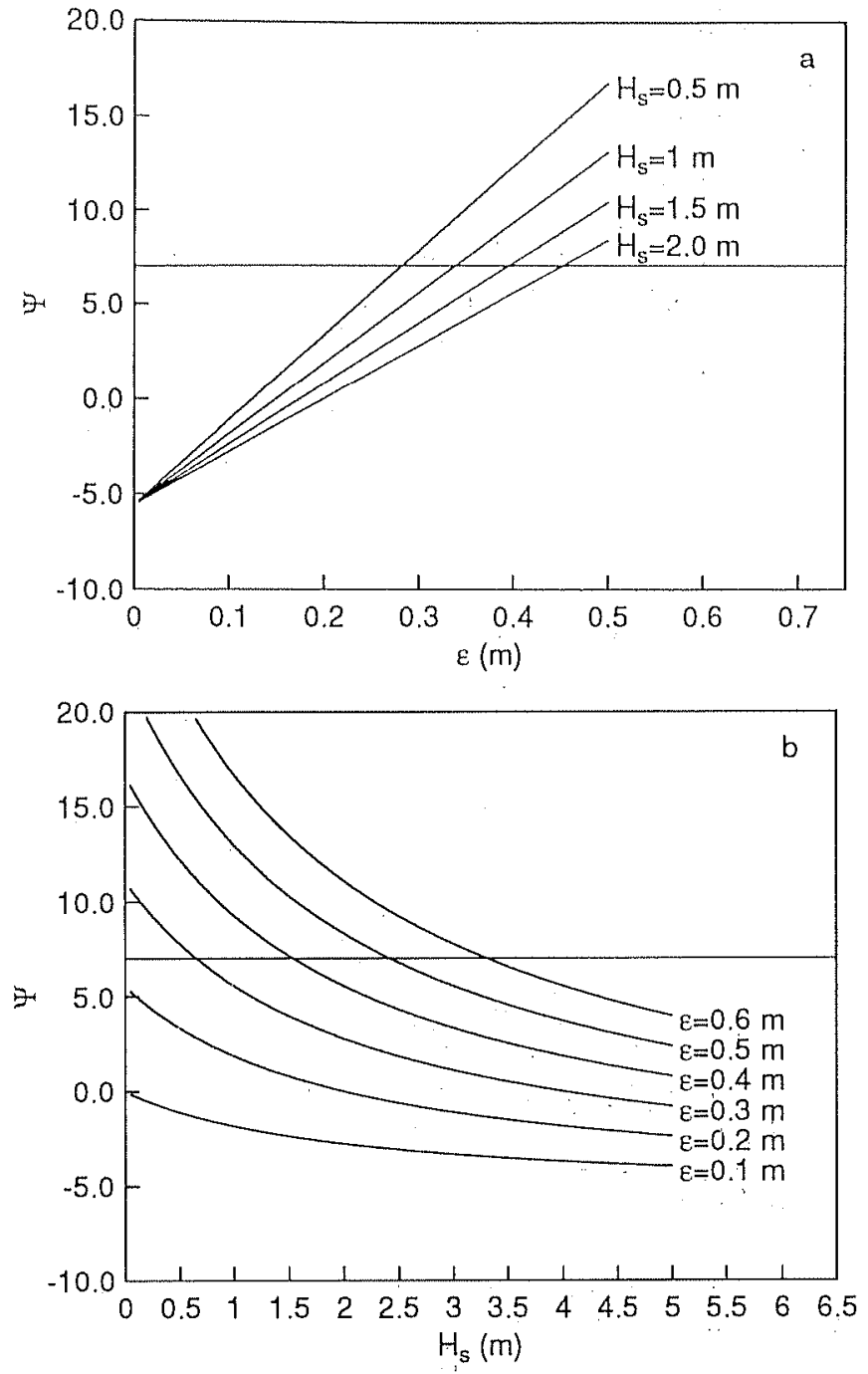

Fig. 3. Log-likelihood ratio $\Psi^{\prime}(a)$ as function of $\varepsilon$ for four different $H_{\mathrm{s}}$ values and $(b) \Delta H_{\text {s }}$ for six different $\varepsilon$ values. The solid lines indicate the threshold $S$ used for the detection.

level is higher for the satellite estimates than for the in situ ones, it can be expected that $\varepsilon$ (estimated as the difference between two consecutive $\dot{H}_{\mathrm{s}}$ estimates) will also be higher. It can be seen from Figure 3 that the algorithm might thus detect a rupture for the satellite data and not for the in situ data. This will result in the detection of shorter stationary low sea states from the satellite data than from in situ data. Furthermore, it can also be seen in Figure 3 that as the noise level is higher for $0<H_{\mathrm{s}}<1.5 \mathrm{~m}$ than for $1.5 \mathrm{~m}<H_{\mathrm{s}}<3 \mathrm{~m}$, the algorithm might be unstable. For example, if one considers an $\varepsilon$ of $0.4 \mathrm{~m}$, detection will occur for $H_{\mathrm{s}}=1.5 \mathrm{~m}$ (see Figure $3 \mathrm{~b}$ ), and if one considers an $\varepsilon$ of $0.3 \mathrm{~m}$, detection will occur for $H_{\mathrm{s}}=0.75 \mathrm{~m}$. Great care must thus be taken when interpreting stationarity for low sea states.

For both locations, Frigg and Palanca, the results of the detection algorithm were used to build two stationary sea state data sets: the $\left(H_{\text {stat }}, T_{\text {stat }}\right)$ ensemble of the sea states stationary in time and the $\left(H_{\text {stat }}, L_{\text {stat }}\right\}$ ensemble of the sea states stationary in space. To insure consistency between the in "situ and the satellite $H_{\mathrm{s}}$ measurements, the Geosat altimeter data were considered in a box surrounding the location. The size of the box is a compromise between the local sea state conditions and the minimum length of the $H_{s}$ altimeter records necessary for detection of the stationary state. Previous studies [Tournadre and Ezraty, 1990] have shown

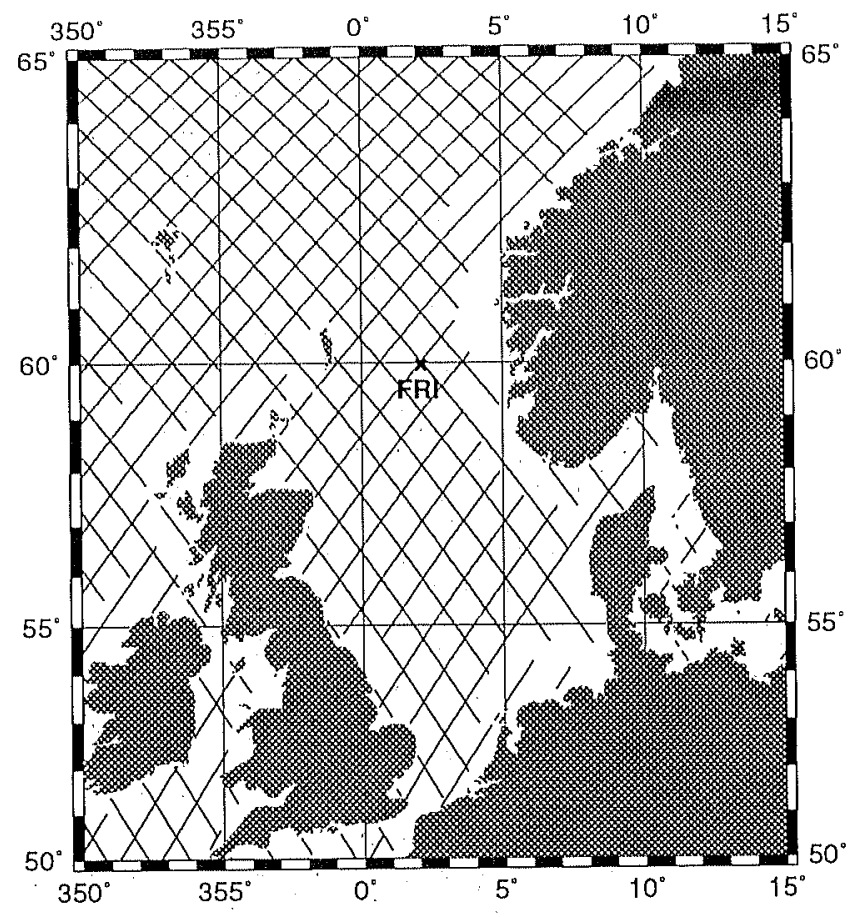

Fig. 4. Geosat ground tracks near Frigg (FRI) during one cycle of the Exact Repeat Mission (17-day repeat cycle).

that the $H_{\mathrm{s}}$ statistics are consistent for about $200 \mathrm{~km}$ around Frigg and $300 \mathrm{~m}$ around Palanca. The stationary state detection algorithm was thus performed on all the Geosat altimeter records located within a $10^{\circ} \times 10^{\circ}$ box around Frigg and within a $15^{\circ} \times 15^{\circ}$ box around Palanca. Data from cycles 1 to 43 (from November 6, 1986 to November 8,1988 ) of the Exact Repeat Mission were used for the Frigg analysis and data from cycles 1 to 63 (from November 6 ,

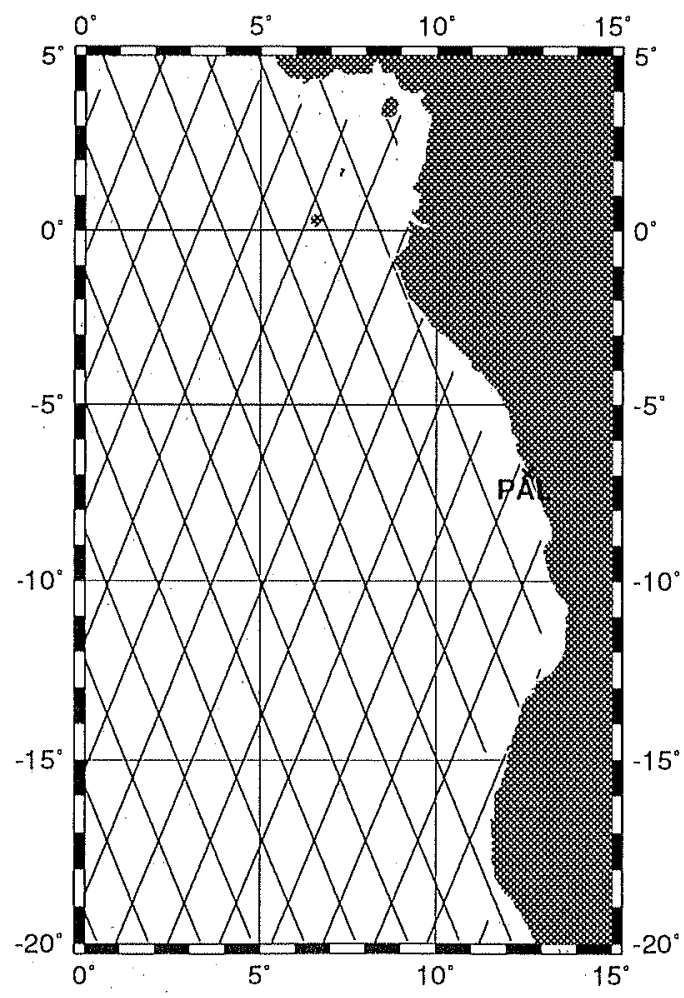

Fig. 5. Geosat ground tracks near Palanca (PAL) during one cycle of the Exact Repeat Mission. 
1986 to September 13, 1989) were used for the Palanca analysis. Figures 4 and 5 present the Geosat ground tracks near Frigg and Palanca, respectively.

A detailed statistical analysis of the four stationary states ensembles is presented in the appendix. This analysis reveals that for both locations, the height of stationarity $\left(\mathrm{H}_{\text {stat }}\right)$ is distributed according to a Gumbel law in both time and space and that the duration $\left(T_{\text {stat }}\right)$ and the length $\left(L_{\text {stat }}\right)$ of stationarity present a very large dispersion and are exponentially distributed. Because of this very large dispersion, no scale can be considered as predominant. In both cases, the duration and length distribution present very similar nondimensional statistical characteristics. As expected from what is know about the Palanca and Frigg wave climate (see appendix), the mean duration and length of stationarity are more than twice as large at Palanca ( 6 hours and $189 \mathrm{~km}$ ) as at Frigg ( 3 hours and $63 \mathrm{~km}$ ).

In the Frigg case, where wind waves are predominant, the heights and duration of stationarity as well as the height and the length can be considered as statistically independent, and no relation exists between the energy level $\left(H_{\text {stat }}\right)$ and the duration and length of stationarity. In the Palanca case, where swell predominates, the length and duration of stationarity decrease when the energy increases (see appendix).

\section{SPECTRAL ANALYSIS}

To complete the stationarity analysis of the significant wave height time and space series a spectral analysis was performed for both locations. Stationarity analysis can also be viewed as a low pass filter and can be used to filter the high-frequency noise (geophysical and/or instrumental) and to isolate the low-frequency component of the $H_{\mathrm{s}}$ variations. In contrast to curve fitting (such as cubic spline functions) used in previous studies [Challenor et $a l ., 1986]$ to approximate the low-frequency signal, the stationarity analysis can be considered to have some physical meaning.

The approximation of the $H_{\mathrm{s}}$ (in time or space) by the stationary states can be expressed as follows: there exists real numbers $\left\{t_{i}\right\}_{i=1, \ldots, N}$ a partition of $[0, \mathrm{~T}]$ and $\left\{a_{i}\right\}_{i=1, \ldots, N}$ such that

$$
H_{\mathrm{s}}(t)=\sum_{i=1}^{N} a_{i} \times \Pi_{\Delta t_{i}}\left(t-t_{0}\right)+\varepsilon(t)=H_{\mathrm{st}}(t)+\varepsilon(t)
$$

where the $\Pi_{\Delta}$ are rectangular window functions of width $\Delta t_{i}=\left(t_{i+1}-t_{i}^{i}\right) / 2$ centered at $t_{0}=\left(t_{i+1}+t_{i}\right) / 2, \varepsilon$ is a Gaussian noise $(N(0, \sigma))$ and $H_{\text {st }}$ is the stationary component of the signal. Each rectangular window is a low-pass filter whose cutoff frequency depends on the window width and on the time delay. Indeed, the Fourier transform of the window function $\Pi_{\Delta t_{i}}\left(t-t_{0}\right)$ is defined by

$$
T F\left(\Pi_{\Delta t_{i}}\left(t-t_{0}\right)\right)=2 \Delta t_{i} \operatorname{sinc}\left(2 \pi \Delta t_{i} v\right) \exp \left(-2 \pi i t_{0_{i}} v \Delta t\right)
$$

The cutoff frequency of the stationarity analysis will depend on the mean width of the windows, i.e., the mean duration (or length) of stationarity. Here, $\varepsilon(t)$ can be considered as consisting of geophysical and/or instrumental noise. The spectral analysis was performed on the raw data $\left(H_{\mathrm{s}}\right)$ and on the low-frequency signal $\left(H_{\mathrm{st}}\right)$ as well as on the residual $(\varepsilon)$.

To insure consistency with the time analysis, only the Geosat records located within a $15^{\circ} \times 15^{\circ}$ box centered at Frigg or Palanca were considered. The Geosat ground tracks of data were then broken into contiguous data records of 200 points for Frigg and 300 points for Palanca. The 200-point limit for Frigg was imposed by the width of the North Sea. Similarly, to allow comparison of the temporal and spatial spectral characteristics, particularly the spectral slopes, the time series of $H_{\mathrm{s}}$ data were cut into 200-samplelong records for Frigg and 300-sample-long records for Palanca. The stationary state detection algorithm was then applied to each record using the noise parameters presented in Table 1 . The results were used to compute $H_{\text {st }}$ and the noise $\varepsilon$ (equation (7)). Then a discrete Fourier transform was perform on each data record, and the results were squared to produce an estimate of the energy spectrum as a function of the frequency $v$ or the spatial wavenumber $k$.

Before examination of the average energy spectra, the spectra $E\left(k_{i}\right)$ or $E\left(v_{i}\right)$ for each data record were scaled by the variance to produce normalized energy spectra $E_{N}\left(k_{i}\right)$ or $E_{N}\left(v_{i}\right)$ or variance spectra $\left(k_{i}\right.$ and $v_{i}$ are the $i^{\text {th }}$ wavenumber and frequency band, respectively). The normalized energy spectrum is defined by the following relation:

$$
E_{N}\left(k_{i}\right)=\frac{E\left(k_{i}\right)}{\sum_{i} E\left(k_{i}\right)}
$$

where $E_{N}\left(k_{i}\right)$ is the normalized spectral density in the wavenumber (or frequency) band $i, E\left(k_{i}\right)$ is the raw spectral density in band $i$, and the sum in the denominator is the overall variance. Elimination of the variance in the spectral characteristics allows comparison of spectral shapes from individual realizations. The normalized spectra were then ensemble averaged to produce a mean spectrum. This spectrum possesses a high statistical reliability, having $2 N$ degrees of freedom (dof) if $N$ is the number of records. An individual spectrum has $\sim 2$ dof [Bendat and Piersol, 1971; Båth, 1974].

\subsection{Time Analysis}

Figure $6 a$ presents the normalized spectra for Frigg. A total of 84 records' were used for the computations. As $H_{s}$ is sampled ev-
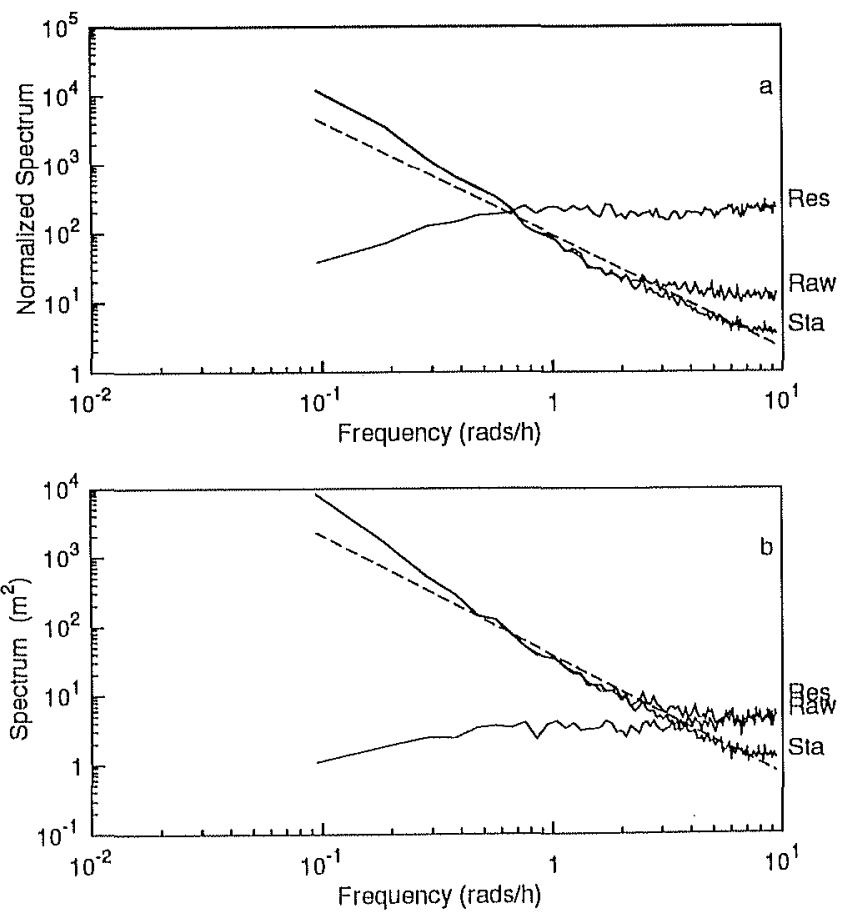

Fig. 6. Spectral analysis in time of the Frigg in situ data from January 1, 1984, to December 31, 1984. (a) Average normalized Spectra of $H_{\mathrm{s}}$ (Raw), $H_{s t}$ (Sta), and residual $\varepsilon$ (Res) (see Equation (9)). (b) Average unnomalized Spectra of $H_{s}$ (Raw), $H_{\mathrm{st}}$ (Sta), and residual $\varepsilon$ (Res). The dashed lines show the mean square fit of the $H_{s t}$ spectra. 
TABLE 2.Slope of the Mean Normalized and Unnormalized $H_{31}$ and $H_{s}$ Spectra for the Different Data Sets

\begin{tabular}{lcccccc}
\hline \multirow{2}{*}{ Data Set } & $\begin{array}{c}\text { Degrees } \\
\text { of }\end{array}$ & \multicolumn{2}{c}{ Stationary Data } & & \multicolumn{2}{c}{ Raw Data } \\
\cline { 3 - 4 } \cline { 6 - 7 } & Freedom & Normalized & Unnormalized & & Normalized & Unnormalized \\
\hline Frigg in Situ & 168 & -1.65 & -1.74 & & -1.21 & -1.28 \\
Frigg Satellite & 1166 & -1.72 & -1.75 & & -1.39 & -1.52 \\
Palanca in Situ & 106 & -1.69 & -1.69 & & - & - \\
Palanca Satellite & 1378 & -1.69 & -1.63 & & -1.21 & -1.08 \\
\hline
\end{tabular}

ery $20 \mathrm{~min}$ and as the record length is 66.67 hours ( 2.8 days), the frequencies that can be examined range from $9.5 \mathrm{radh}^{-1}$ to $0.0935 \mathrm{rad} \mathrm{h}^{-1}$. As it can be expected from the hypothesis made in the detection algorithm on the distribution of the noise $\varepsilon$, its spectrum is almost flat, denoting a white noise. This shows the good quality of the filtering using the stationary state detection algorithm. The $H_{\text {st }}$ spectrum is very nearly linear when plotted in a $\log$-log space. The spectrum appears to follow a simple power law dependence on frequency. The least squares estimate of the spectral slope is -1.65 (see Table 2). The raw spectral slope is close to 1.21 ,

Figure $6 b$ presents the unnormalized spectra for Frigg. The shape of these spectra is similar to that of the normalized ones. The estimated slope of the $H_{\text {st }}$ spectrum is -1.74 . Comparison of the $H_{s}$ and $H_{\text {st. }}$ spectra shows that the filtering is important for frequencies over $2 \mathrm{rad} \mathrm{h}^{-1}$, i.e., for periods over 3.2 hours which is roughly the mean duration of stationarity for Frigg.

The normalized spectra estimated for Palanca are presented in Figure $7 a$, fifty-three 300-sample-long records were considered. The frequencies range from $9.5 \mathrm{rad} \mathrm{h}^{-1}$ to $0.063 \mathrm{rad} \mathrm{h}^{-1}$. The noise level is important for high frequencies and may result from a low number of dof. The $H_{\text {st }}$ spectrum presents a power law depen-
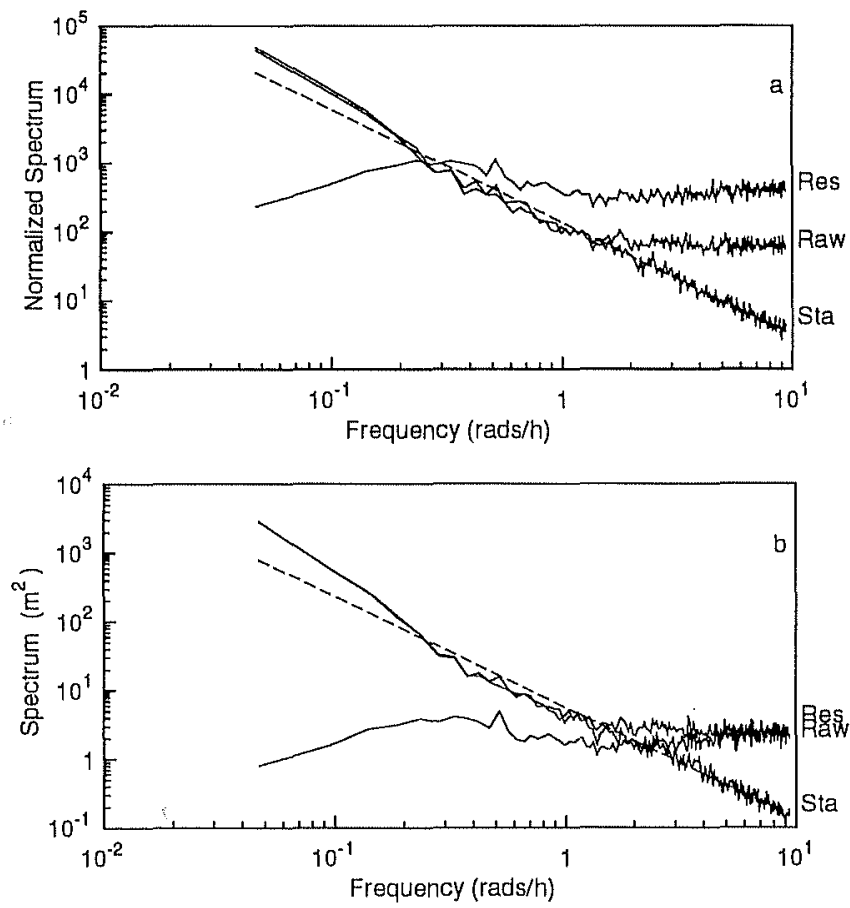

Fig. 7. Spectral analysis in time of the Palanca in situ data from November 1, 1984, to November, 1, 1985. (a) Average normalized Spectra of $H$ (Raw), $H_{s t}$ (Sta), and residual $\varepsilon$ (Res). (b) Average unnormalized Spectra

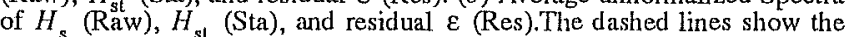
mean square fit of the $H_{\text {st }}$ spectra. dence on the frequency. The estimated slope is -1.69 . Figure $7 b$ presents tie unnormalized spectra. The slope of the $H_{\text {st }}$ spectrum is -1.69 . The filtering is important for frequencies over $1 \mathrm{rad} \mathrm{h}^{-1}$, i.e., for periods over 6.3 hours. This value is close to the mean duration of stationarity. The $H_{\mathrm{s}}$ spectrum exhibits a high level of high-frequency noise, and the dependence on the frequency does not follow the same power law for all frequencies.

\subsection{Space Analysis}

As the resolution is $\sim 7 \mathrm{~km}$ and as the length of the record is $1400 \mathrm{~km}$ ( $\sim 2000 \mathrm{~km}$ for Palanca), the wavelengths that can be examined range from $\sim 14 \mathrm{~km}$ to $1400 \mathrm{~km}(2000 \mathrm{~km})$. The average normalized energy spectra for $H_{\mathrm{s}}, H_{\text {stat }}$, and $\varepsilon$ for Frigg are presented in Figure $8 a$. A total of 583 data records were used for the computation. As compared with the time analysis, the space spectra, having a higher number of dof are smoother. Regardless, the shapes of the spectra are very similar in both domains and the $H_{\text {st }}$ spectrum is very nearly linear when plotted on a log-log scale. The spectrum appears to follow a simple power law dependence on
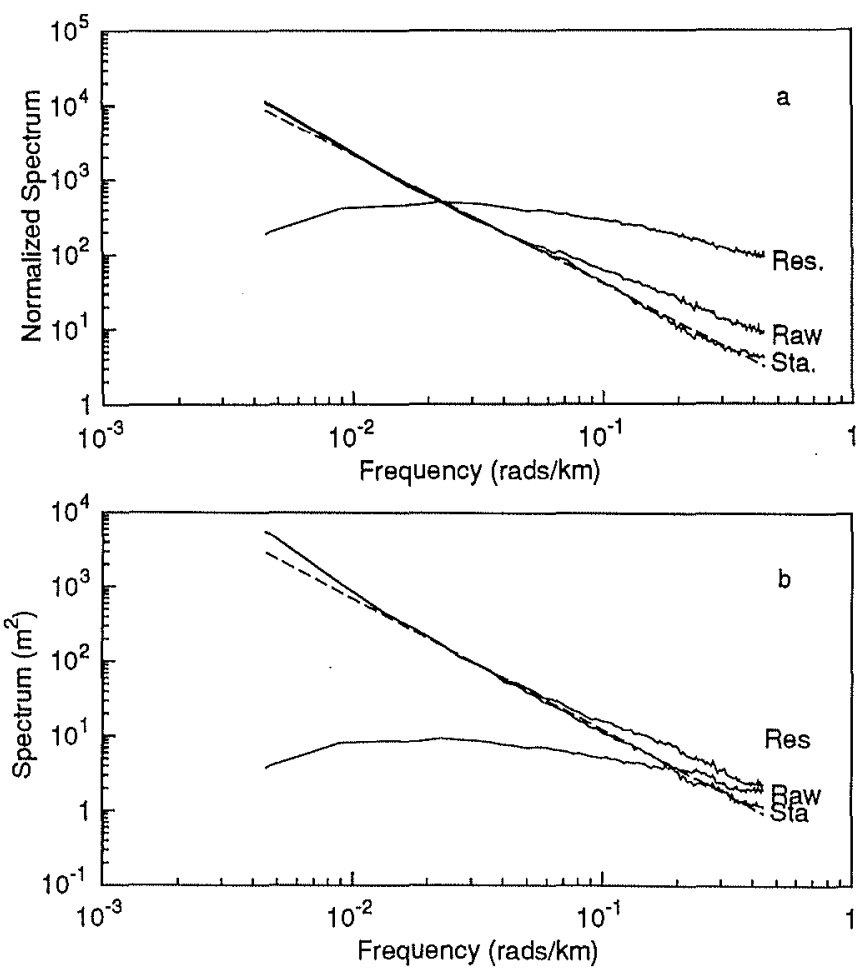

Fig. 8. Spectral analysis in space of the Geosat data records for cycles 1 to 43 (from November 6, 1986, to November 6, 1988) located in a $15^{\circ} \times 15^{\circ}$ box centered at Frigg. (a) Average normalized Spectra of $H_{s}$ (Raw), $H_{\text {st }}$ (Sta), and residual $\varepsilon$ (Res). (b) Average uniormalized Spectra of $H_{\mathrm{s}}$ (Raw), $H_{s}$ (Sta), and residual $\varepsilon$ (Res). The dashed lines show the mean square fit of the $H_{\text {st }}$ spectra. 

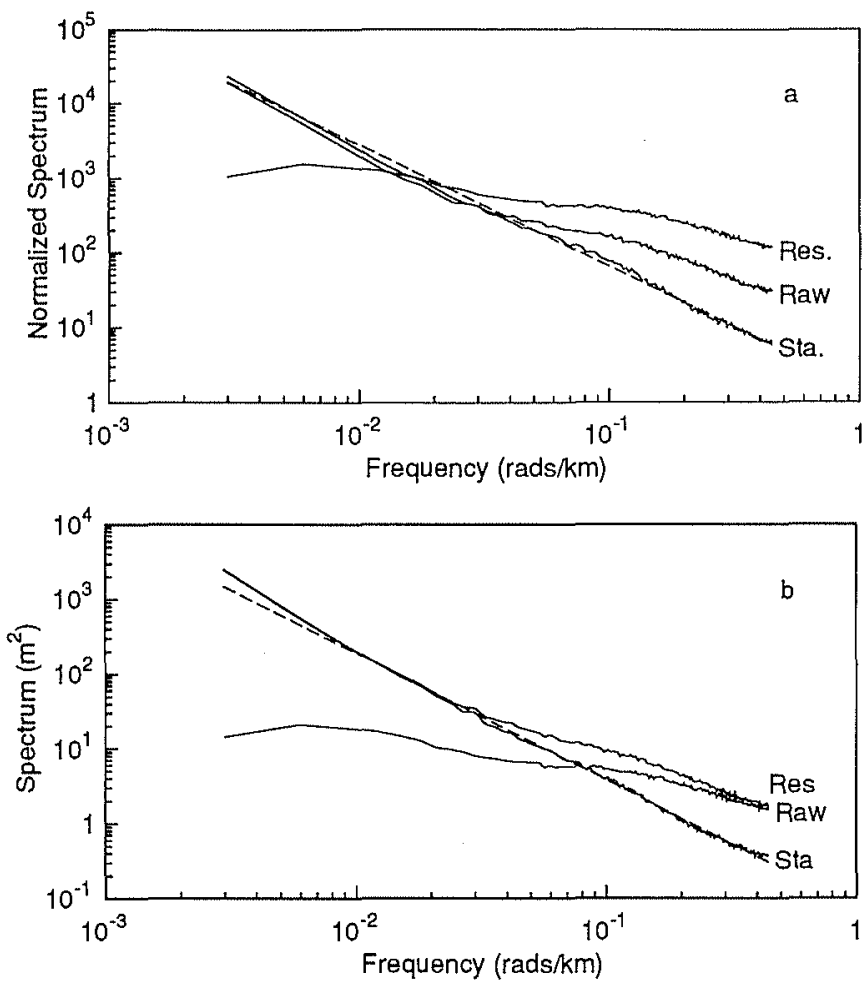

Fig. 9. Spectral analysis in space of the Geosat data records for cycles 1 to 62 (from November 6, 1986, to September 13,1989) located within a $15^{\circ} \times 15^{\circ}$ box centered at Palanca. (a) Average normalized Spectra of $H_{s}$ (Raw), $H_{\text {st }}$ (Sta), and residual $\varepsilon$ (Res), (b) Average unormalized Spectra of $H_{\mathrm{s}}$ (Raw), $H_{\mathrm{st}}$ (Sta), and residual $\varepsilon$ (Res). The dashed lines show the mean square fit of the $H_{\text {st }}$ spectra.

wavenumber. The least squares estimate of the spectral slope is 1.72 , to be compared with -1.65 for the time spectral slope. For the raw spectrum of $H_{\mathrm{s}}$, the slope is close to -1.39 . This value is almost identical to the one presented by Monaldo [1990], i.e., -1.3867 for a similar analysis.

Figure $8 b$ presents the unnormalized energy spectra. The shapes of the normalized and unnormalized spectra are very similar, and the slopes are very close. The comparison of the $H_{\mathrm{s}}$ and $H_{\mathrm{st}}$ spectra shows that the filtering is important for wavenumbers greater than $0.1 \times 10^{-1} \mathrm{Tad} \mathrm{km}^{-1}$, i.e., approximately the inverse of the mean length of stationarity. The slope is -1.75 , which is very close to the value found for the normalized spectrum and to the value of the time spectral slope (-1.74).

An identical analysis was performed for Palanca. A total of 689 data records were considered. Figure $9 a$ presents the normalized spectra. As compared with the time analysis, the high-wavenumber noise $\left(>1 \times 10^{-1} \mathrm{rad} \mathrm{km}^{-1}\right)$ in the space domain is lower than the high-frequency noise $\left(>2 \mathrm{rad} \mathrm{h}^{-1}\right)$. This might result from the large difference in number of dof between the time and space spectra (see Table 2). As in the case of Frigg, the $H_{\mathrm{st}}$ spectrum is almost linear when plotted on a log-log scale, indicating a power law dependence on the wavenumber. The mean square estimate of the slope is -1.63 . The raw spectrum $\left(H_{\mathrm{s}}\right)$ slope estimate is close to 1.21. Figure $9 b$ presents the average spectra (unnormalized). They are similar in shape to the normalized ones. The estimate of the $H_{\text {st }}$ spectral slope is -1.69 .

\subsection{Comparison of Time and Space Analysis}

For both locations the spectral analysis shows that the filtered spectra present a simple power law dependance on the frequency or the wave number. For Frigg the slopes of the $H_{\text {st }}$ time and space spectra are -1.74 and -1.75 , respectively, and for Palanca, 1.69 and -1.69 . It appears that the power laws are almost identical in time and space. This suggests a linear dispersion relation for $H_{\mathrm{s}}$. Furthermore, for Frigg, the time and space spectra are respectively

$$
\begin{aligned}
& S(v)=1.56 v^{-1.74} \\
& S(k)=0.007 k^{-1.75}
\end{aligned}
$$

The value of the slope is close to the $-5 / 3$ of the enstrophy cascade of the two dimensional isotropic turbulence. If we suppose isotropy in space, if we consider a first order approximation that $S(v)=S(k)$ (even if the time periods considered for the two analyses are different) and if we assume that the dispersion relation is of the form $v=\mathrm{C}_{g} k$ where $\mathrm{C}_{g}$ is the wave group velocity (because $H_{\mathrm{s}}$ is related to the energy of the waves), we find a group velocity of $6.2 \mathrm{~m} \mathrm{~s}^{-1}$. As the water depth is about $100 \mathrm{~m}$ at Frigg and as the mean wave period, computed from the sea-surface elevation data, is $7.7 \mathrm{~s}$, the deep water approximation can be considered as valid for the propagation of the observed waves. In this approximation, the phase velocity is $12.4 \mathrm{~m} \mathrm{~s}^{-1}$. The water depth at Frigg is $100 \mathrm{~m}$. In a first order approximation, the deep water propagation relation, $\mathrm{T}=2 \pi \mathrm{C} / \mathrm{g}$, can be used to compute a mean $H_{\mathrm{s}}$ period. We find a period of $7.6 \mathrm{~s}$ which is very close to the 7.7-s period computed from the sea surface elevation data at Frigg in 1984.

For Palanca, the spectra are respectively

$$
\begin{aligned}
& S(v)=0.218 v^{-1.69} \\
& S(k)=0.003 k^{-1.69}
\end{aligned}
$$

The slopes are identical, which suggests a linear dispersion relation. However, it is difficult to estimate a mean $H_{\mathrm{s}}$ period because the water is only $47 \mathrm{~m}$ deep at Palanca. The computation of the mean period using the same method as for the Frigg case gives $5 \mathrm{~s}$, to be compared with a mean period of $9.7 \mathrm{~s}$ computed from the in situ data. For a period of $9.7 \mathrm{~s}$ the critical depth for the deep water approximation is $73 \mathrm{~m}$ (using the $d / \lambda=0.5$ criterion where $\mathrm{d}$ is the water depth and $\lambda$ is the wavelength). At this depth, the deep water approximation is no longer valid at least for long sea states such as long swell. The effect of depth on the phase and group velocities being nonlinear in respect to the period, an estimate of the mean period using a linear dispersion relation cannot be valid.

\section{SUMMARY AND DISCUSSION}

Altimeter measurements and in situ data from radar distance meter have been used to determine the temporal and spatial variability of significant wave height $\left(H_{\mathrm{s}}\right)$ on scales from 1 hour to 3 days and $20 \mathrm{~km}$ to $1500 \mathrm{~km}$. Previous studies [Queffeulou, 1983; Challenor et al., 1986] have shown that the $H_{\mathrm{s}}$ variations in both time and space can be split into a low-frequency part, associated with meteorological synoptic events, and a high-frequency noise component, instrumental and/or geophysical. They have also shown that there exist short distances over which the statistical characteristics of the $H_{\mathrm{s}}$ process change rapidly, i.e., where $H_{\mathrm{s}}$ is no longer stationary. This Jeads us to a scale analysis that combines stationarity and correlation analysis.

The stationarity analysis is based on the method proposed by Lavielle and Rio [1987] and Labeyrie [1990]. A sea state is defined as a stationary state of a piecewise stationary random wave process. A sequential method for change detection in the $H_{\mathrm{s}}$ process is used to identify the stationary states which are defined by their energy $\left(H_{\mathrm{s}}\right)$ and their duration or length of stationarity. Four data 
sets were analyzed: two of intensive in situ measurements (one $H_{\mathrm{s}}$ estimate every $20 \mathrm{~min}$ ) and two of Geosat altimeter measurements, corresponding to two locations, one in the North Sea $\left(60^{\circ} \mathrm{N}, 2^{\circ} \mathrm{E}\right)$ and one in the equatorial Atlantic $\left(6^{\circ} \mathrm{S}, 12^{\circ} \mathrm{E}\right)$.

For both locations the stationary analysis shows that in both time and space the duration and length of stationarity have large dispersion and are exponentially distributed. No scale can thus be considered as predominant. The duration and length of stationarity present very similar nondimensional statistical characteristics, indicating identical behavior in both the time and space domains. As expected, from what is known about the wave climate in the North Sea and in the equatorial Atlantic, the mean duration and length of stationarity are more than twice as large for the equatorial location ( 7 hours $20 \mathrm{~min} ., 189 \mathrm{~km}$ ) as for the North Sea location ( 2 hours 50 min., $63 \mathrm{~km}$ ). For the North Sea location, where the wave climate results mainly from the action of the wind, $H_{\mathrm{s}}$ and the duration of stationarity, as are stochastically independent $H_{\mathrm{s}}$ and the length of stationarity. For the equatorial Atlantic location, where the wave climate is dominated by the propagation of swell, the duration or length of stationarity decreases as the energy increases. In the doldrums regions, low sea states are associated with long periods of very calm weather and thus with long time or length of stationarity. Higher sea states result from either swell propagation or convective events.

The stationary state detection can also be view as a low-pass filter and can be used to isolate the low-frequency component of $H_{\mathrm{s}}$ associated with meteorological events. The low-frequency signal is defined as the stationary component of $H_{\mathrm{s}}$. The spectra of the residual, i.e., the difference between the raw $H_{s}$ and the low-frequency signal, are almost flat, denoting white noise and showing the good quality of the filtering.

The spectral analysis of both in situ and altimeter data sets and of both filtered and raw $H_{\mathrm{s}}$ records reveals that the spectra of the filtered $H_{\mathrm{s}}$ have similar shapes in the time and space domains for both locations. They also have similar shapes in the two locations. They are found to obey simple power law dependencies on frequency or wavenumber. In the North Sea, spectra in time and space decayed approximately as $k^{-1.74}$, while in the equatorial Atlantic the falloff is a slightly less steep $k^{-1.69}$. Comparison of the raw and filtered spectra shows that the filtering is important for periods and wavelength greater than the mean duration or length of stationarity. The spectral slopes found for the raw spatial spectra are very similar to the one presented by Monaldo [1990] for Seasat $H_{\mathrm{s}}$ data record.

The near equality of the temporal and spatial spectral slope suggests a mean linear relation of dispersion for the $H_{s}$ propagation at mesoscale, i.e. for the propagation of potential energy at the seasurface. Assuming isotropy in space, it is possible to estimate a mean wave group velocity, using the deep water approximation for the wave propagation it is then possible to compute a mean wave phase velocity and thus a mean wave period. For the North Sea lo- cation, where the water depth permitted the use of the deep water approximation, the estimated mean wave period was $7.6 \mathrm{~s}$. This value is very close to the $7.7 \mathrm{~s}$ found from in situ measurements.

The slopes of the filtered spectra are close to the $-5 / 3$ turbulence cascade. However, the interpretation of significant wave heights in terms of turbulence is not easy. A possible way to investigate this behavior of $H_{s}$ could be the following. As the waves results mainly from the wind action, $H_{\mathrm{s}}$, i.e., the potential energy, could be viewed as a tracer of the wind. By analogy with passive tracer such as temperature in the atmosphere, the wave propagation could be interpreted as a diffusive process. Under these hypotheses, using turbulence models, it is possible to relate the tracer spectrum to the wind kinetic energy spectrum. For example, in two-dimensional homogeneous isotropic turbulence, the theory predicts that a $k^{-5 / 3}$ law for a passive tracer in the inverse -energy-cascade range of the wind where the kinetic energy spectrum follows a $k^{-5 / 3}$ law [Lesieur and Herring, 1985]. This theory is attractive for the interpretation of the $H_{\mathrm{s}}$ spectra: however, the results found so far on the wind variability at mesoscale do not confirm the theory of two-dimensional turbulence models for the wind, especially in the inverse-energy-cascade range. Indeed, using Seasat scatterometer data, Freilich and Chellon [1986] found a wind kinetic energy wavenumber dependence of the order of $k^{-2}$ for wavelengths from 200 to $2200 \mathrm{~km}$. Refined models of near-surface atmospheric turbulence, better knowledge of air-sea interaction, and comparison of wind and $H_{s}$ spectra will be necessary in order to understand the $H_{s}$ space and time variability.

\section{APPENDIX: STATISTICAL ANALYSIS OF THE STATIONARY STATE ENSEMBLES}

This appendix presents the statistical analysis of the $\left\{H_{\text {stat }}, T_{\text {stat }}\right\}$ and $\left\{H_{\text {stat }}, L_{\text {stat }}\right\}$ ensemble of the sea states stationary in time and space, respectively, for the Frigg and Palanca locations Table A1 presents the statistical characteristics of the four data sets, i.e., the mean value $\left(\overline{H_{\text {stat }}}\right.$ and $\overline{T_{\text {stat }}}$ or $\left.\overline{L_{\text {stat }}}\right)$, the standard deviation $\sigma$, the skewness Skew, and the kurtosis Kur, i.e. the third and fourth order non-dimensional central moments.

\section{A1. Frigg}

A1.I. Heights and duration of stationarity. Figure A1 presents the histograms of ( $a$ ) the height (i.e., energy) and of $(b)$ the duration. The high value of the kurtosis found for the duration shows that the distribution has very large dispersion and that the duration is exponentially distributed. This can be easily seen in Figure A1. The exponential probability density function (pdf) is of the form

$$
\begin{array}{ll}
P(x)=\frac{1}{a} \exp \left(-\frac{x-u}{a}\right) & x>u \\
P(x)=0 & x<u
\end{array}
$$

For such a distribution, the mean value is $a-u$ and the standard deviation is $a$.

\begin{tabular}{|c|c|c|c|c|c|c|c|c|}
\hline \multirow[b]{2}{*}{ Data Set } & \multicolumn{4}{|c|}{$H_{\text {stat }}$ Data Set } & \multicolumn{4}{|c|}{$T_{\text {stat }}$ or $L_{\text {stat }}$ Data Set } \\
\hline & $\begin{array}{c}\overline{H_{\text {stat }}}, \\
\mathrm{m}\end{array}$ & $\begin{array}{c}\sigma_{H} \\
\mathrm{~m}\end{array}$ & Skew & Kur & $\overline{T_{\text {stat }}} / \overline{L_{\text {stat }}}$ & $\sigma$ & Skew & Kur \\
\hline Frigg in Situ & 2.4 & 1.41 & 0.90 & 3.42 & 2 hours, $50 \mathrm{~min}$ & 2 hours, $45 \mathrm{~min}$ & 2.38 & 11.36 \\
\hline Frigg Satellite & 1.9 & 1.22 & 1.10 & 4.16 & $63 \mathrm{~km}$ & $71 \mathrm{~km}$ & 2.57 & 11.70 \\
\hline Palanca in Situ & 1.3 & 0.40 & 0.56 & 2.88 & 7 hours, $20 \mathrm{~min}$ & 8 hours, $31 \mathrm{~min}$ & 2.66 & 12.51 \\
\hline Palanca Satellite & 1.3 & 0.34 & 0.86 & 4.18 & $189 \mathrm{~km}$ & $252 \mathrm{~km}$ & 2.20 & 12.23 \\
\hline
\end{tabular}

TABLE Al, Statistical Characteristics of the Temporal and Spatial Stationary Data Sets 

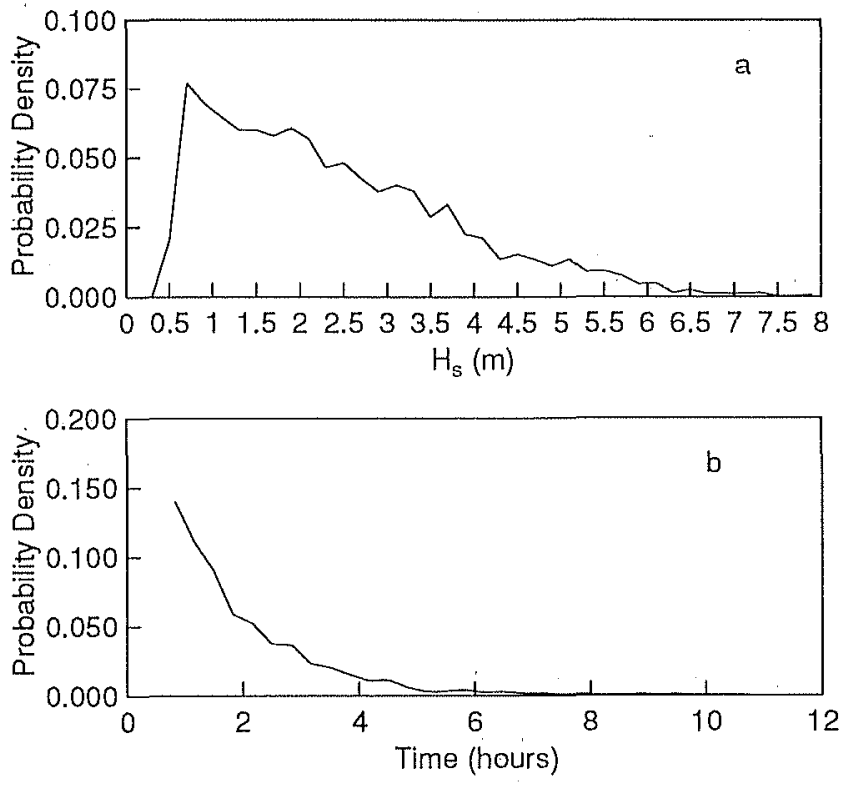

Fig. A 1. Histogranis of $(a)$ height $H_{\text {sint }}$ and (b) time of stationarity $T_{\text {st }}$ for Frigg, in situ data. Temporal analysis from November $1,1984^{\text {stat }}$ to December 31, 1984 (2681 sea states).

Because of the very large dispersion of the distribution, no duration can be considered as predominant. It should be noted that the mean duration of stationarity is close to 3 hours, which is generally considered as the time of stationarity of a sea state in mid-latitudes. The lower bound for $T_{\text {stat }}$ is $20 \mathrm{~min}$, which is the length of the recording time. The concept of stationarity as defined in the previous section cannot be analyzed for shorter records with current data.

The statistical characteristics of the height distribution show that it is distributed according to a Gumbel pdf of the form

$$
\begin{array}{ll}
P(x)=\frac{1}{a} \exp \left(-\frac{x-u}{a}\right) \exp \left(-\exp \left(-\frac{x-u}{a}\right)\right) & x>u \\
P(x)=0 & x<u
\end{array}
$$

The mean value for a Gumbel probability density function is $0.5722 a+u$ and the standard deviation is $\pi a / 6$.

The dependence (or independence) of $H_{\text {stat }}$ and $T_{\text {stat }}$ can be studied by the conditional expectation function (cef), i.e., the mean value of one parameter as a function of the other. Figure A2 presents the cef of the duration given the time (Figure A2a) and the cef of the height given the duration (Figure A2b). As can be seen, the cef of the duration and height are almost constant. This indicates that no relationship exists between the energy level of a sea state and its duration of stationarity.
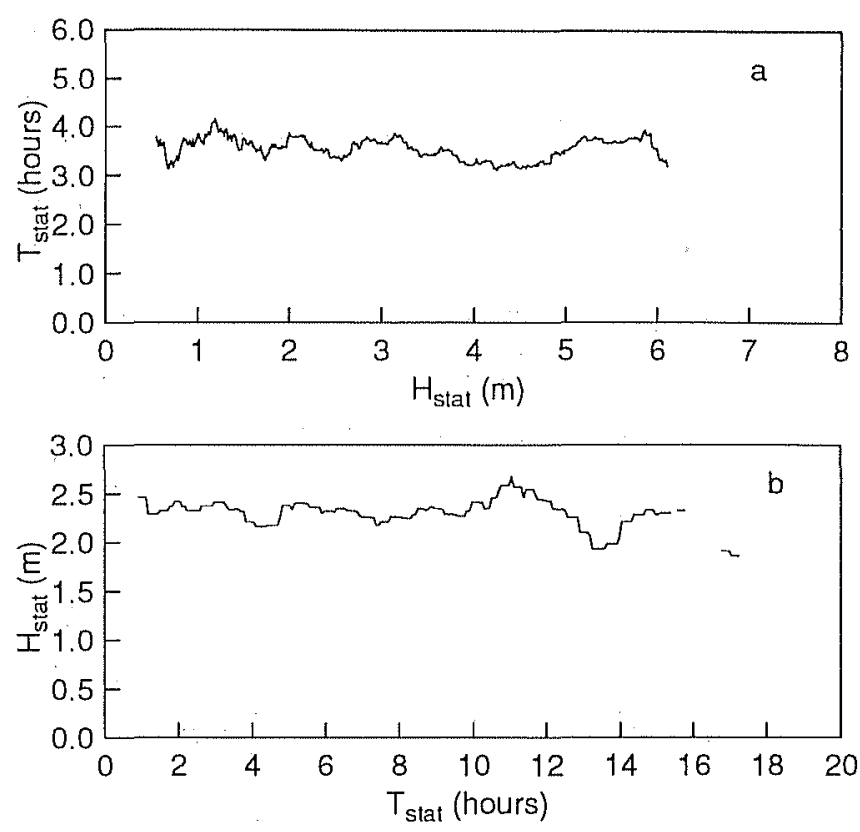

Fig. A 2. Conditional expectation function $(a)$ of $H_{\text {stat }}$ and $(b)$ of $T_{\text {stat }}$ for Frigg in situ stationary states; 2861 sea states.

Another way to study the interdependence of $H_{\text {stat }}$ and $T_{\text {stat }}$ is to analyze the probability laws of $H_{\text {stat }}$ and $T_{\text {stat }}$ for different classes of $T_{\text {stat }}$ and $H_{\text {stat }}$. Tables A2 and A3 present the coefficients of the mathematical laws fitting the empirical probabilities (i.e. the cumulative histograms) of $H_{\text {stat }}$ and $T_{\text {stat }}$ for different classes of $T_{\text {stat }}$ and $H_{\text {stat }}$. The probability of $T_{\text {stat }}$ is modeled by exponential laws, and the probability of $H_{\text {stat }}$, by Gumbel laws. The modeling was done using the method presented by Labeyrie [1985]. The distribution of $H_{\text {stat }}$ is independent of the duration of the sea state. The distribution of the duration can also be considered as independent of the height. The coefficients of the exponential laws are almost constant, except for the last class, which contains few sea states $(<100)$. This shows that $H_{\text {stat }}$ and $T_{\text {stat }}$ are statistically independent.

Figure $A 3$ presents the mean of the raridom jumps $\left(\Delta H_{\mathrm{s}}\right)$ between consecutive sea states given as a function of $H_{\mathrm{s}}$. The standard deviation $\sigma$ (i.e., the noise level) is also presented in the figure and is computed from the classical estimator

$$
\sigma=\sqrt{\frac{1}{n-1} \sum_{i=1}^{n}\left(H_{s_{i}}-H_{\text {stat }}\right)^{2}}
$$

\begin{tabular}{|c|c|c|c|c|c|c|c|}
\hline \multirow{2}{*}{ Data Set } & \multirow{2}{*}{ Parameler } & \multirow{2}{*}{ Coefficient } & \multicolumn{5}{|c|}{$H_{\text {stat }}$ Class, m } \\
\hline & & & $0-2$ & $2-4$ & $4-6$ & $\geq 6$ & Toual \\
\hline \multirow{2}{*}{ Frigg in Situ } & \multirow{2}{*}{$T_{\text {slat }}$} & 4 & -0.5 & -0.7 & -0.4 & -0.5 & -0.7 \\
\hline & & $a$ & 3.3 & 3.4 & 3.0 & 2.3 & 3.2 \\
\hline \multirow{2}{*}{ Frigg Satellite } & \multirow{2}{*}{$L_{\text {stat }}$} & $u$ & -4.5 & 1.7 & 8.7 & 8.5 & 4.0 \\
\hline & & $a$ & 81 & 82 & 87 & 87 & 82 \\
\hline \multirow{2}{*}{ Palanca in Situ } & \multirow{2}{*}{$T_{\text {stat }}$} & $u$ & -3.0 & -7.0 & -2.2 & - & -3.6 \\
\hline & & $a$ & 12.3 & 7.8 & 3.0 & - & 7.0 \\
\hline \multirow{2}{*}{ Palanca Satellite } & \multirow{2}{*}{$L_{\text {stat }}$} & $u$ & -249 & -115 & -174 & - & -168 \\
\hline & & $a$ & 123 & 247 & 225 & - & 137 \\
\hline
\end{tabular}

TABLE A2. Coefficients of the Pareto Laws Modeling the Probability of $T_{\text {stat }}$ or $L_{\text {stat }}$ for Different $H_{\text {stat }}$ Classes and for the Four Different Data Sets 
TABLE A3. Coefficients of the Gumbel Laws Modeling the Probability of $H_{\text {stat }}$ for Different $T_{\text {stat }}$ or $L_{\text {stat }}$ Classes

\begin{tabular}{|c|c|c|c|c|c|c|c|}
\hline \multirow{2}{*}{ Coefficient } & \multicolumn{7}{|c|}{ Classes } \\
\hline & 1 & 2 & 3 & 4 & 5 & 6 & Total \\
\hline \multicolumn{8}{|c|}{ Frigg in Situ } \\
\hline$T_{\text {stat }}$, hours & $0-2$ & $2-4$ & $4-6$ & $6-8$ & $8-10$ & $>10$ & \\
\hline$u$ & 1.7 & 1.7 & 1.6 & 1.7 & 1.7 & 1.7 & 1.8 \\
\hline$a$ & 1.1 & 1.1 & 1.1 & 1.2 & 1.1 & 1.1 & 1.0 \\
\hline \multicolumn{8}{|c|}{ Frigg Satellite } \\
\hline$L_{\text {stat }}, \mathrm{km}$ & $0-21$ & $21-42$ & $42-63$ & $63-84$ & $84-105$ & $>105$ & \\
\hline$u$ & 1.2 & 1.5 & 1.7 & 1.8 & 1.8 & 1.8 & 1.3 \\
\hline$a$ & 1.0 & 1.1 & 1.0 & 1.1 & 0.9 & 0.9 & 1.0 \\
\hline \multicolumn{8}{|c|}{ Palanca in Situ } \\
\hline$T_{\text {stat }}$, hours & $0-3$ & $3-6$ & $6-9$ & $9-12$ & $12-15$ & $>15$ & \\
\hline$u$ & 1.3 & 1.1 & 1.0 & 1.0 & 1.0 & 0.9 & 1.1 \\
\hline$a$ & 0.3 & 0.3 & 0.3 & 0.3 & 0.2 & 0.2 & 0.3 \\
\hline \multicolumn{8}{|c|}{ Palanca Satellite } \\
\hline$L_{\text {stat }}, \mathrm{km}$ & $0-21$ & $21-42$ & $42-63$ & $63-84$ & $84-105$ & $>105$ & \\
\hline$u$ & 0.9 & 1.0 & 1.0 & 1.1 & 1.2 & 1.3 & 1.0 \\
\hline$a$ & 0.5 & 0.4 & 0.4 & 0.4 & 0.4 & 0.4 & 0.4 \\
\hline
\end{tabular}

It can be seen in the figure that the $\Delta H_{\mathrm{S}}$ and $\sigma$ variations are almost linear. The mean square linear fits for these two parameters are

$$
\Delta H_{\mathrm{s}}=0.08 H_{\mathrm{s}}+0.05 \quad \sigma=0.04 H_{\mathrm{s}}-0.02
$$

These values are close to the ones chosen as input to the detection algorithm which shows the stability of the detection algorithm.

A1.2. Heights and lengths of stationarity. Figure A4 presents the $H_{\text {stat }}$ and $L_{\text {stat }}$ histograms. As for the time analysis, the height and length are distributed according to a Gumbel and exponential distributions, respectively. No length can therefore be considered as predominant. It should be noted that the nondimensional statistical characteristics of the $L_{\text {stat }}$ and $T_{\text {stat }}$ ensembles are very similar (see Table A1). This indicates the same kind of behavior in the time and space domains.

The cef of $H_{\text {stat }}$ given the length of stationarity is approximately constant (see Figure A5). The cef of $L_{\text {stat }}$ is almost constant for $H_{\text {stat }}$ greater then $1.5 \mathrm{~m}$. For lower sea states the mean length of stationarity is lower. This results from the $H_{\mathrm{s}}$ noise behavior which leads to the instability of the detection algorithm (see section 4). Tables A2 and A3 present the coefficients of the probabili-

(B) $\Delta \mathrm{H}=0.047+0.085{ }^{*} \mathrm{H}_{\mathrm{s}}$

(A) $\sigma=-0.018+0.044{ }^{*} H_{s}$

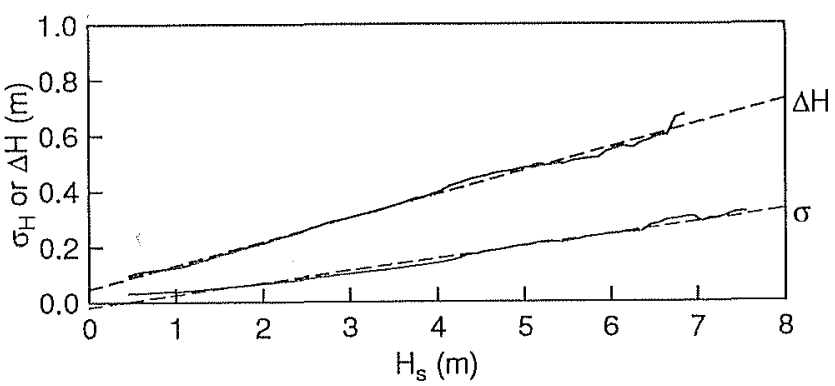

Fig. A 3. Mean jumps $\left(\Delta H_{s}\right)$ and variance $\sigma_{H}$ for the Frigg temporal stationary state analysis. ty law (estimated from the cumulative histograms) for $H_{\text {stat }}$ and $L_{\text {stat }}$ for different classes of $L_{\text {stat }}$ and $H_{\text {stat }}$. From Table A2, it can be seen that the standard deviation within each class is almost constant. The variation of $u$ reflects the variation of the mean $T_{\text {stat }}$ value. Thus except for low sea states, the distribution of $L_{\text {stat }}$ is quite independent of $H_{\text {stat }}$. Reciprocally, the $H_{\text {stat }}$ distributions are almost independent of the length of stationarity. This shows the independence of the $H_{\text {stat }}$ and $L_{\text {stat }}$ variables. If $7 \mathrm{~km}$ in space are considered to be equivalent to $20 \mathrm{~min}$ in time (see section 3), then the mean length of stationarity $(63 \mathrm{~km})$ is roughly equivalent to 3 hours, i.e., the mean duration of stationarity. This suggests a linear dispersion relation for the propagation of $H_{\mathrm{s}}$.
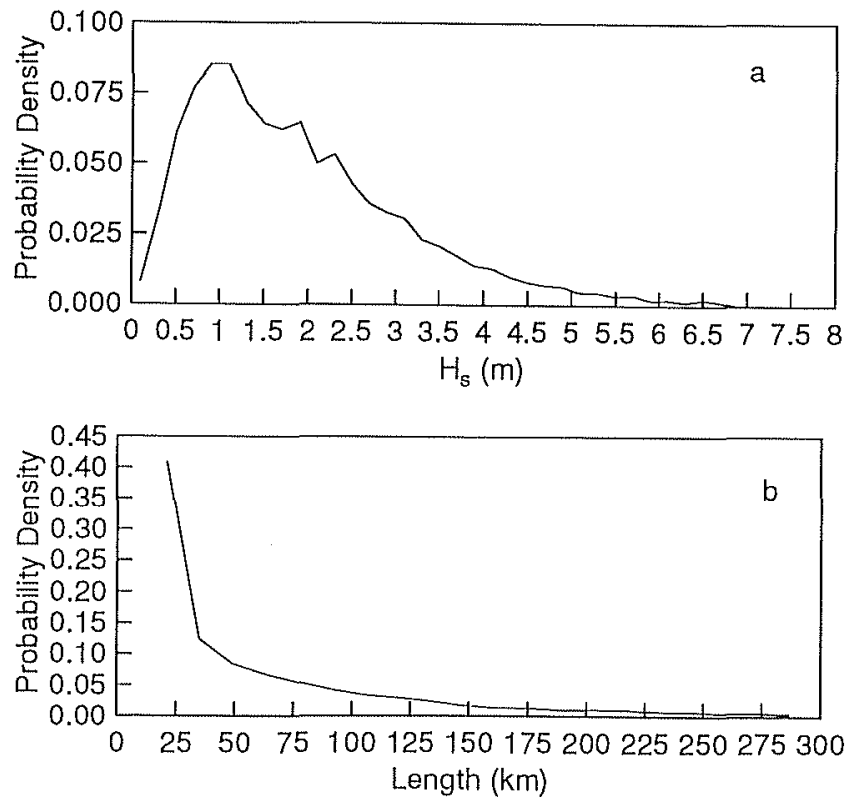

Fig. A 4. Histograms of $(a)$ height $H_{\text {stat }}$ and $(b)$ length of stationarity $L_{\text {stat }}$ for Frigg, satellite data. Space analysis for cycles 1 to 43 of the Geosat Exact Repeat Mission, from November 6, 1986, to Novenber 8, 1988 (8293 sea states). 

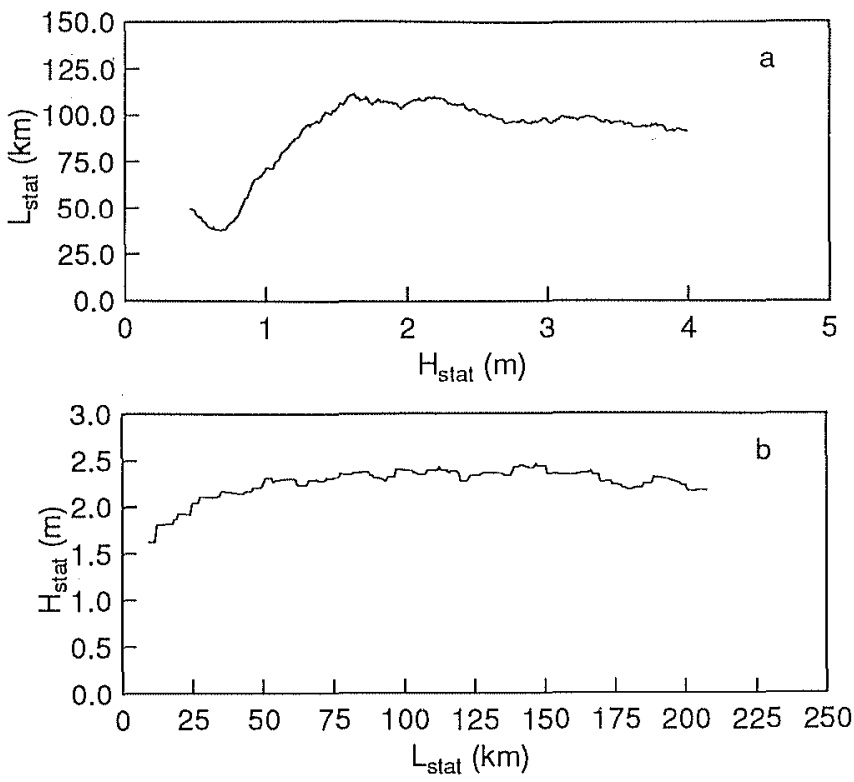

Fig. A 5. Conditional expectation function of $(a) H_{\text {stat }}$ and of $(b) L_{\text {stat }}$ for Frigg spatial stationary states.

\section{A2. Palanca}

Compared to Frigg, the Palanca sea state data set is characterized by the predominancy of swell [Tournadre and Ezraty, 1990] which propagates either from the South Atlantic or from the North Atlantic (see, for example, the study of Cartwright et al. [1977] for Saint Helena Island).

A2.1. Duration and height. Figure A6 shows the height and duration histograms. As it can be expected from what is known about the wave climate in equatorial oceans (swell predominancy), the mean duration of stationarity is more than twice as large as in the Frigg case (see Table A1). However, the nondimensional statistical characteristics of the distribution (skewness and kurtosis) are very close to those found for Frigg. The ciuration is exponentially dis-
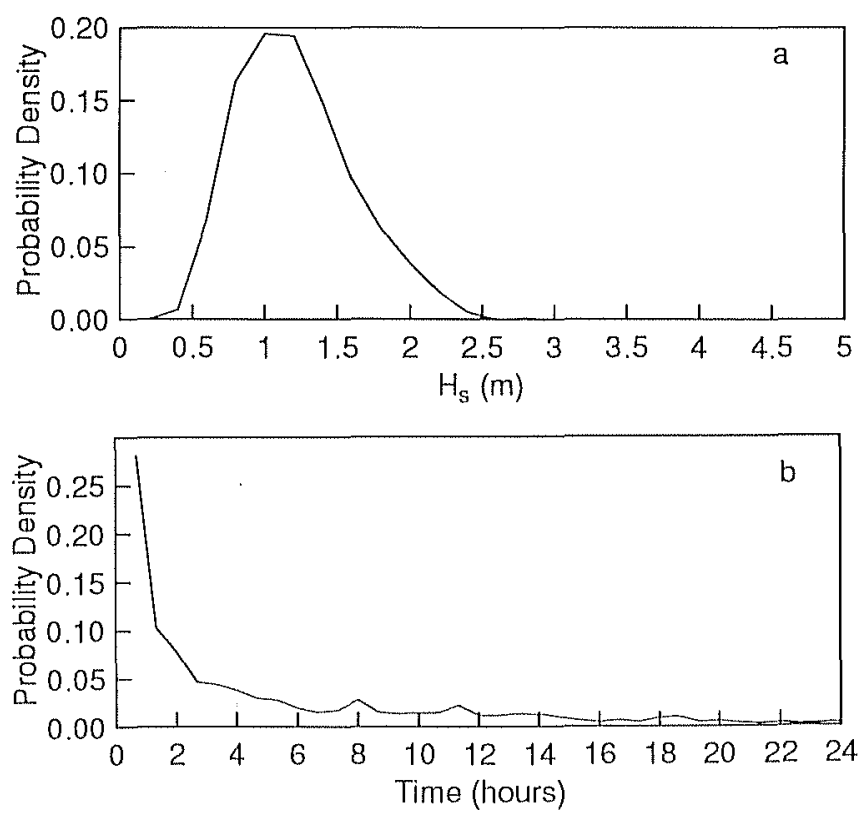

Fig. A 6 . Histograms of $(a)$ height $H_{\text {stat }}$ and $(b)$ time of stationarity $T_{\text {stat }}$ for Palanca, in situ data. Temporal analysis from November 1,1984 , to November 1, 1985 (1449 sea states).
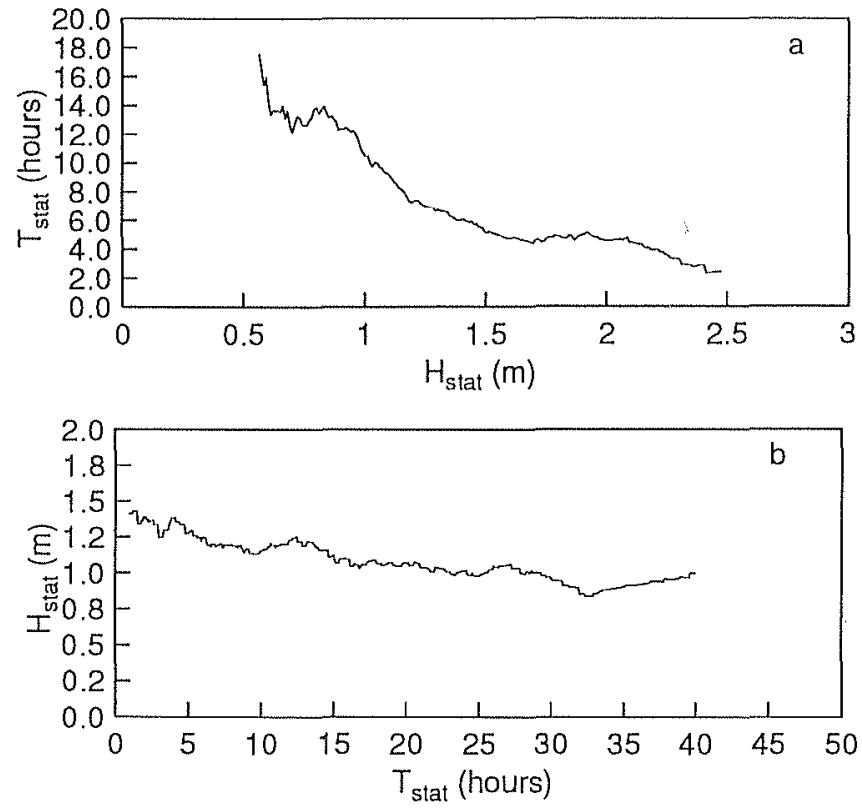

Fig. A 7. Conditional expectation function (a) of $H_{\text {stat }}$ and (b) of $T_{\text {stat }}$ for Palanca in situ stationary states.

tributed and has a large dispersion. The height is distributed according to a Gumbel law.

Figure A7 presents the cef of the duration given the height as well as the cef of the height given the duration. The cef of the duration decreases as the height increases. Palanca being located in the doldrums region, there exist long periods of very calm weather during which the wind is almost null. Low sea states $(<1 \mathrm{~m})$ can therefore be expected to be associated with long duration. Larger sea states $(>1.5 \mathrm{~m})$ are associated with either convective events or swell propagation and can thus be expected to be characterized by shorter duration.

It can be seen in Tables A2 and A3 that the $T_{\text {stat }}$ distribution depends on the energy level, which confirms what has been said pre-
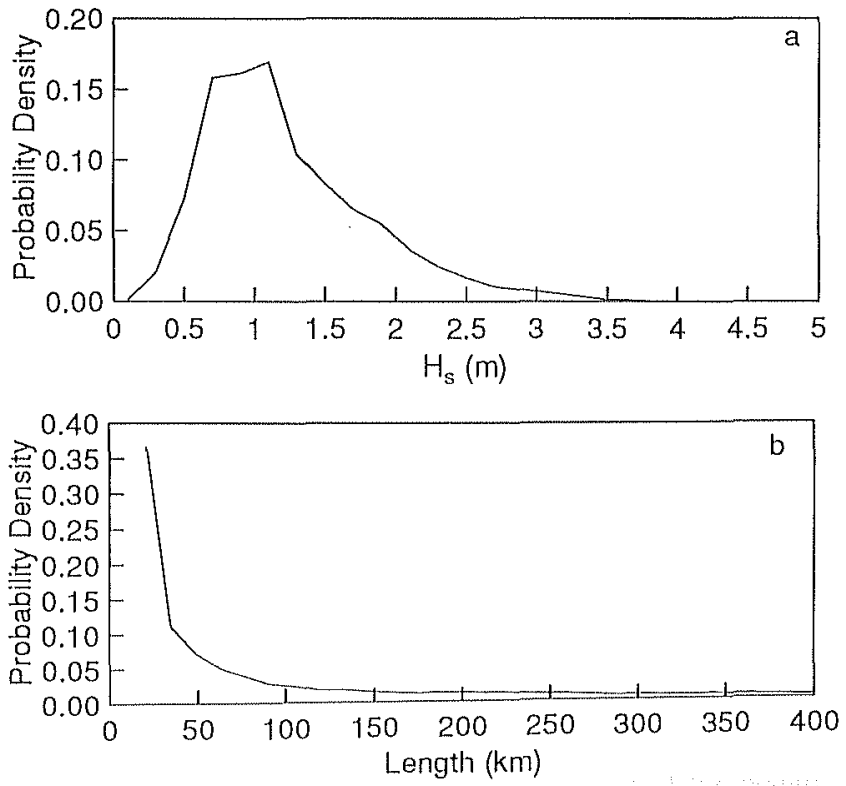

Fig. A 8. Histograms of $(a)$ height $H_{\text {slat }}$ and $(b)$ length of stationarity $L_{\text {stat }}$ for Palanca, satellite data. Space analysis for cycles 1 to 62 of the Geosat Exact Repeat Mission from November 6, 1986, to September 13, 1989 (11052 sea states). 

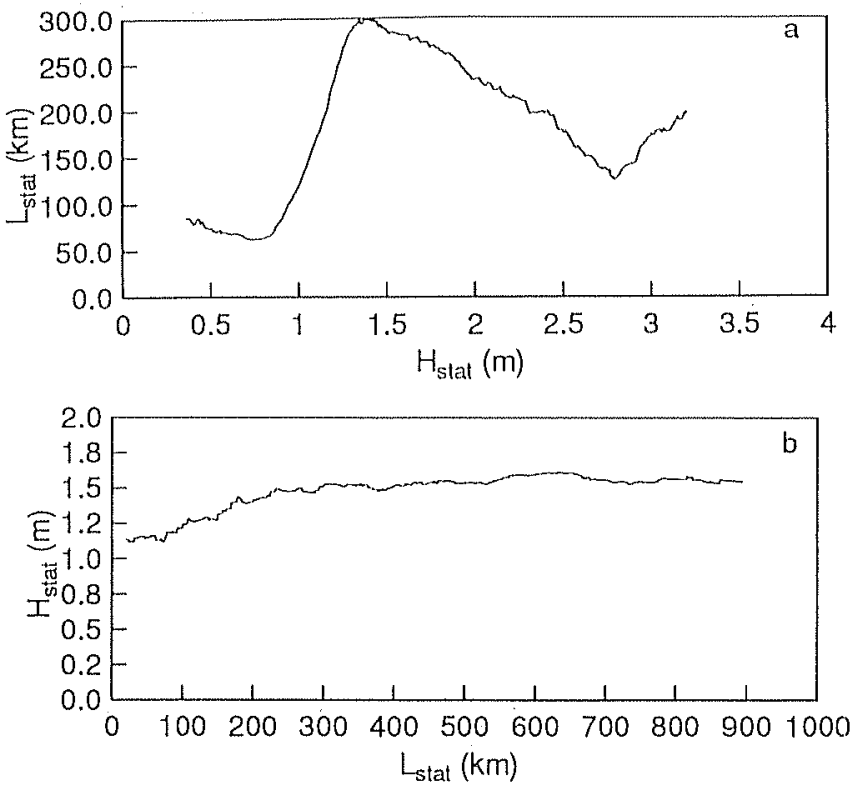

Fig. A 9. Conditional expectation function (a) of $H_{\text {stat }}$ and (b) of $L_{\text {stat }}$ for Palanca satellite stationary states.

viously. The $H_{\text {stat }}$ distribution is less dependent on the duration of stationarity.

A2.2. Length and height. Figure A8 presents the height and length histograms. The length is exponentially distributed and the height follows a Gumbel law. The mean length of stationarity is almost 3 times larger than that found in the Frigg case. Some sea states are stationary over more than a thousand kilometers. They can be associated with the propagation of swell trains. Figure A9 shows the cef of the length given the height and the cef of the height given the length. For sea states greater than $1 \mathrm{~m}$, the length of stationarity decreases as the height increases. As for Frigg, low sea states $(<1 \mathrm{~m})$ are associated with short lengths of stationarity. This results again from an instability of the detection method because of a high noise level for low sea states.

Acknowledgments. We are indebted to $\mathrm{M}$. Ollagnon and A. Robin for processing the in situ data sets used in this study. We thank K. Katsaros, B. Chapron, and P. Queffeulou for helpful comments on the manuscript.

\section{REFERENCES}

Basseville, $M_{.}$, Detecting changes in signals and systems : A survey, Automatica, 24, 309-326, 1988.

Basseville, M. and A. Benveniste, Design and comparative study of some sequential jump detection algorithms for digital signals, IEEE Trans. Acoust. Speech. Signal Process., ASSP-31, 521-534, 1983.

Bath, M., Spectral Analysis in Geophysics, Elsevier, Amsterdam, 1974.

Bendat, J.S., and A.G. Piersol, Random Data: Analysis and Measurement Procedures, Wiley-Intersciences, New York, 1971.

Brown, G.S., Estimation of surface wind speed using satellite borme radar measurements at normal incidence, J. Geophys. Res., 84, 3974-3978, 1979.
Cartwright, D.E., J.S. Driver, and J.E. Tranter, Swell waves at Saint Helena related to distant stoms, Q.J. R. Meteorol. Soc, 103, 655-683, 1977.

Challenor, P.G., Spatial variation of significant wave height, Satellite Microwave Remote Sensing, 451-460, Ellis Horwood, Chischester, England, 1983.

Challenor, P.G., T.H. Guymer, and M.A. Srokosz, The influence of spatial and temporal scales on calibration/validation, in ERS-1 Wind and Wave Calitbration Workshop, 17-26, European Space Agency, Noordwijk, Netherlands, 1986.

Colc, T.D., and C. May, Geosat- $\Lambda$ data users/ground system interface control document (ICI), Tech. Rep. 7292-9510, Johns Hopkins Univ., Laurel, Md, May 1985.

DeCambry, O., and J. Labeyrie, Optimisation d'une mesure de l'encrassement d'un échangeur par détection de ruptures dans un modèle, Proceeding 10eme Colloque sur le Traitement du signal et ses Applications, Nice, France, May 1985:

Elf Aquitaine Norge,, Frigg Field environmental conditions report, Rep. 253/82, Société Nationale Elf Aquitaine, Páu, France, Nov. 1981.

Fedor, L.S., and G.S. Brown, Wave height and wind speed measurements from the Scasat radar altimeter, J. Geophys. Res., 87, 3254-3260, 1982.

Freilich, M.H., and D.B. Chelton, Wavenumber spectra of Pacific winds measured by Seasat scatterometer, J. Phys. Oceanogr., 16, 741-757, 1986.

Labeyrie, J., ST.EX.OM: A statistical package for the prediction of extreme values, Proceeding 4th. International Conference on Engineering Software, London, 17-32, Springer-Verlag, Ncw-York, 1985.

Labeyrie, J., Stationary and transient states of random sea, J. Mar. Struct. Des. Constr. Safety., 3, 43-58, 1990.

Labeyrie, J., Time scales and statistical uncertainties in the prediction of extreme environmental conditions, J, Reliability Eng. Syst. Safety, 31, $1-24,1991$

Lavielle, M., and E. Rio, Identification d'états de mer stationnaires ou de transition par détection de ruptures d'un modèle, Publ, LEMPAB $N^{\circ} 1$, p. 44, LEMPAB-IFREMER, Brest, France, June 1987.

Lesieur, M., and J. Herring, Diffusion of a passive scalar in twodimensional turbulence, J. Fluid Mech., 161, 77-95, 1985.

Longuet-Higgins, M.S., Ori the statistical distribution of the heights of sea waves, J.Mar. Res., 11, 245-266, 1952.

McArthur, J.L., P.C. March, and J.S. Wall, The Geosat radar altimeter, Johns Hopkins APL Tech. Dig., 8, 176-181, 1987.

Monaldo, F., Expected differences between buoy and radar altimeter estimates of wind speed and significant wave height and their implications on buoy-altimeter cómparisons, J. Geophys. Res., 93, 2285-2302, 1988.

Monaldo, F., Corrected spectra of wind speed and significant wave height, J. Geophys. Res., 95, 3399-3402, 1990.

Queffeulou, P., Seasat wave height measurement: A comparison with sea-truth data and a wave forecasting model - Application to the geographic distribution of strong sea states in storms, J. Geophys. Res, 88 , $1779-1788,1983$.

Tournadre, J., and R. Ezraty, Local climatology of wind and sea state by means of satellite radar altimeter measurements, J. Geophys. Res., 95 , $18,225-18,268,1990$.

Toumadre, J., and A. Robin, Analyse des mesures altimétriques et in situ de hauteur significative des vagues à Frigg. Détection des ruptures, Doc. Trav. DRO-EO, DIT, IFREMER, Brest, France, 1990.

Willsky, A.S., and A.L. Jones, A generalized likelihood ratio approach to the detection and estimation of jumps in linear systems, IEEE Trans. Autom. Control, 21, 108-112, 1976.

J. Toumadre, IFREMER, Centre de Brest, BP 70, 29280 Plouzané, France.

(Received March 7, 1992; revised September 16, 1992 accepted October 14, 1992.) 